\title{
Vibrational convection in a heterogeneous binary mixture. Part II. Frozen waves
}

\author{
Anatoliy $\operatorname{Vorobev}^{1} \dagger$, and Tatyana Lyubimova ${ }^{2,3}$ \\ ${ }^{1}$ Department of Engineering and Physical Sciences, University of Southampton, SO17 1BJ UK \\ ${ }^{2}$ Institute of Continuous Media Mechanics, Ural Branch RAS, Perm, Russia \\ ${ }^{3}$ Perm State University, Russia \\ (Received xx; revised xx; accepted xx)
}

The action of high-frequency vibrations on a heterogeneous binary mixture that fills in a closed container is numerically modelled to validate the theoretical model obtained in the first part of the work, and to investigate the role of interfacial stresses in the evolution of miscible boundaries. Only weightlessness conditions are considered. A recent experimental study reports the threshold ignition of the frozen waves at a miscible interface even under weightlessness conditions, which cannot be explained on the basis of the classical approach that represents a binary mixture as a single phase fluid with impurity. This effect, however, can be well explained on the basis of the phase-field equations that are derived in the first part of our work. In particular, we found that when the vibrational forcing is sufficiently strong (the vibrational forcing is primarily determined by the amplitude of the vibrational velocity), above a certain threshold value, then the interface becomes shaped into a 'frozen' (time-independent to the naked eye) structure of several pillars (the frozen waves) with the axes perpendicular to the directions of vibrations. The threshold level of vibrations is determined by the interfacial stresses that need to be associated with miscible interfaces. The time needed for setting up the frozen pattern is relatively small, determined by hydrodynamic processes, however this time grows exponentially near the threshold. The frozen pattern remains stable either indefinitely long (if liquids are partially miscible) or until the interface becomes invisible due to diffusive smearing (if liquids are miscible in all proportions). A further increase of the vibrational forcing alters the number of the pillars, which happens discretely when the intensity of the vibrations surpasses a sequence of further critical levels. Correlation of the results with the previous experimental and theoretical studies validate the new approach making it a useful tool for tracing thermo- and hydrodynamic changes in heterogeneous mixtures.

\section{Introduction}

If a heterogeneous fluid system (a fluid system with interfaces) is subjected to highfrequency vibrations it exhibits a quite peculiar behaviour (see figure 1). For instance, the Rayleigh-Taylor instability may become suppressed by a vibrational forcing that is enforced in the direction perpendicular to the interface. The translational oscillations in a plane that is parallel to the interface may induce a frozen (stationary for the naked eye) relief on the boundary between two liquids (both liquids perform high-frequency oscillatory movements but the interface with a particular shape remains frozen to the naked eye). This effect was first experimentally observed by Wolf (1961, 1970), and was later studied in experiments by Bezdenezhnyh et al. (1991); Talib et al. (2007); Yoshikawa

$\dagger$ Email address for correspondence: A.Vorobev@soton.ac.uk 
(a)

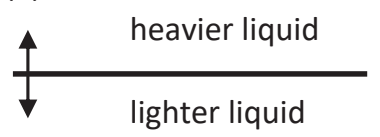

(b)

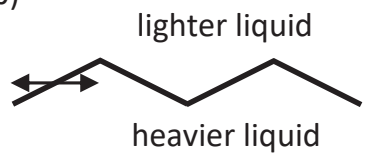

FIGURE 1. Schematic illustrations of the time-averaged effects of high-frequency vibrations on the dynamics of a liquid/liquid interface. (a) The vibrations of fluids in the direction perpendicular to the interface may stabilise the Rayleigh-Taylor instability (keeping a heavier liquid above a lighter liquid). (b) The vibrations along the interface may induce the frozen (time-independent) wave-patten.

\& Wesfreid (2011b), and more recently by Gandikota et al. (2014a,b); Lyubimova et al. (2017) who studied the frozen waves in near-critical hydrogen using the magnetic field to counter-balance the gravity (thus investigating the frozen waves under normal and reduced gravity). First results on the theoretical description (the linear and weakly nonlinear theories, that predict the stability thresholds and shapes of the frozen interfaces, as well as the numerical solution of the full equations for tracing the growth of unstable modes) are summarised in the book by Lyubimov et al. (2003). Later, Talib et al. (2007); Yoshikawa \& Wesfreid (2011a); Lyubimov et al. (2016) and Lyubimov et al. (2017) studied the effects of viscosity and its contrast at interface on the frozen wave instability.

The linear stability analysis developed by Lyubimov \& Cherepanov (1986) for a liquid/liquid interface of an unrestricted size proved that the flat interface becomes unstable when the vibrational forcing exceeds a critical (threshold) level set by expression,

$$
\frac{(\phi a \omega)^{2}}{4}=\frac{\sigma_{*} k}{\rho_{*}}-\frac{\phi g}{k} .
$$

Here, $a$ and $\omega$ are the amplitude and frequency of the vibrations ( $a \omega$ is the amplitude of the vibrational velocity), $k$ is the wavenumber that characterises a disturbance of a flat interface, $g$ is the gravity acceleration, $\sigma_{*}$ is the coefficient of surface tension, $\rho_{*}$ is the typical density (e.g. the average of two densities, $\rho_{1}$ and $\rho_{2}$, that are the densities of two liquids in contact), and $\phi=\left(\rho_{2}-\rho_{1}\right) / \rho_{*}$ is the density contrast. Expression (1.1) is written assuming that $k h>>1$, i.e. the liquid layers are sufficiently deep, and that $\phi<<1$ which is true for most liquid/liquid interfaces (the frozen relief forms when the liquids in contact have comparable densities, and this effect does not exist on e.g. an air/water boundary).

In the current study we are interested in the dynamics of two miscible liquids. The formation of frozen waves (or a frozen relief) on the boundary between two miscible liquids was recently experimentally observed by the research team of V. Shevtsova (see Gaponenko \& Shevtsova 2010; Gaponenko et al. 2015a,b; Shevtsova et al. 2015, 2016). In the experiment, a container with a shape of a rectangular parallelepiped was filled with two miscible liquids, that were taken in equal volumes, and the container was subjected to high-frequency translational vibrations. The experiments were conducted both under normal gravity conditions and under conditions of weightlessness that were achieved by performing the experiments during parabolic flights. The frozen shapes of the liquid/liquid boundaries were photographed and analysed.

The same team conducted the numerical simulations of the experiments (see Gaponenko et al. 2015a,b) by modelling the dynamics of miscible liquids on the basis of a classical approach, when the miscible liquids are represented by a single-phase fluid with impurity, and the liquids are distinguished from each other by concentration levels. Two alternative approaches were used for modelling the influence of the high-frequency 
vibrations: (i) by solving the full equations for a considerably long time period and averaging the flow fields over a time period of vibrations, and (ii) by solving the timeaveraged equations that were earlier derived by Gaponenko et al. (2006) and Gaponenko \& Shevtsova (2010). Both approaches generated very similar results, even when the amplitude of vibrations was not small (in comparison with the size of the container). The numerical results (in particular, the shapes of the frozen wave patterns) were generally in a favorable agreement with with the experimental observations.

Nevertheless, recently, Wolf (2018) noted that the experimental studies by Gaponenko et al. $(2015 a, b)$ do not fully address whether the frozen interfaces remain stable for sufficiently long time periods, as he argues that the frozen structure should become unstable due to development of unstable modes within broaden miscible boundaries.

Another experimental observation that remained poorly understood and that is particularly interesting for our current work is the fact in the experiment the generation of frozen waves occurs when the vibrational forcing is over a certain threshold level (see e.g. Gaponenko et al. 2015a,b), while the classical theory (see equation (1.1)) states that a miscible interface (with no surface tension forces) under weightlessness conditions is unstable to any vibrational forcing (simply because there are no other forces in the equations that may change the shape of a liquid/liquid boundary and may counterbalance the vibrational force). The validity of the linear theory (1.1) was previously confirmed experimentally, including a recent work by Lyubimova et al. (2017). To resolve the discrepancy between the experimental results and theoretical expectations it was proposed to introduce the concept of effective interfacial stresses associated with miscible boundaries. The coefficient of the surface tension was estimated as $\sigma_{*} \sim 6 \cdot 10^{-6} \mathrm{~N} / \mathrm{m}$ for the binary mixture used in the experiment (see Gaponenko et al. 2015b). The concept of surface tension helps to explain the experimental results but at the same time it makes the classical approach that is traditionally used for description of miscible systems, and that completely disregards the interfacial stresses, incapable of describing at least some of the features of frozen waves in miscible systems.

The current study has a two-fold objective. The first aim is to verify the governing equations that are derived in the first part of our work against the available experimental data. The new equations are based on the phase-field approach for the description of a mixture, that, in particular, introduces the effect of dynamic surface tension associated with miscible boundaries (see Joseph \& Renardy 1993; Pojman et al. 2006; Zoltowski et al. 2007; Stevar \& Vorobev 2012; Vorobev 2014). Indeed, it is a common everyday experience that a droplet of honey immersed in tea has a spherical shape, which can only be explained by using the concept of interfacial stresses. When the droplet is fully dissolved in tea, the honey/water interface disappears, and, obviously, the interfacial stresses associated with a miscible interface should also disappear at this moment. In addition, the phase-field approach is based on the extended Fick's law that states that the diffusion flux is proportional to the gradient of chemical potential, not the gradient of concentration. The definition of the chemical potential includes the classical part, that allows for the account of the concentration dependence of the diffusion coefficient, the gravity (barodiffusion) and the surface tension effects.

We would like also to mention that the dissolution of a droplet may be visualized in two different ways. If there are no hydrodynamic motion, then the classical description with a constant diffusion coefficient assumes the liquid/liquid interface remains stationary and the droplet keeps its shape and size while the interface slowly smears until it becomes indistinguishable (invisible). Alternatively, the dissolution may occur through shrinkage of a droplet, so that the interface remains sharp (clearly visible) until the droplet's size becomes zero. The first scenario should be more appropriate for the dissolution process in 
liquids that are miscible in all proportions, while the second scenario is more applicable for partially miscible liquids (which are miscible until the limiting solubilities are reached in each phase). Although, the experiments (see e.g. Zoltowski et al. 2007; Stevar \& Vorobev 2012) suggest that the second scenario works even for fully miscible binary mixtures. The phase-field approach is capable of describing the dissolution of a droplet in accordance with both scenarios.

Thus, the second aim of the current study is to investigate the formation and evolution of the frozen waves on a surface of two (partially-miscible) liquids with the account of the effects of dynamic surface tension, to reveal the role of (quite low) surface tension forces associated with miscible boundaries on the dynamics of binary mixtures.

\section{Problem statement}

In our study we aim to reproduce the settings of the experiments that were fulfilled by Gaponenko et al. $(2015 a, b)$. We assume that two miscible liquids, that are taken in equal volumes and that are initially separated by a thin flat boundary, occupy a closed $2 D$ rectangular container (with the aspect ratio of $2: 1$ ), as shown in Figure $6 a . \dagger$ A container is subjected to translational vibrations along its larger dimension. The weightlessness conditions are assumed.

To facilitate the comparison with other numerical studies, the numerical simulations in our work are fulfilled with the use of two different sets of equations. Both models describe the time-averaged effects that are enforced by high-frequency vibrations (in the sense that a time period of an oscillatory forcing is considerably smaller than typical hydrodynamic time-scales, or $\omega>>\eta_{*} /\left(\rho_{*} L^{2}\right)$ and $\omega>>D_{*} / L^{2}$, where $\eta_{*}$ and $D_{*}$ are the viscosity and diffusion coefficients) on the dynamics of liquid/liquid mixtures (both systems of equations assume that the density contrast is relatively small, $\phi<<1$ ). The first set of equations is derived on the basis of the classical approach, i.e. disregarding the surface tension effects for miscible interfaces and using the Fickian law for description of interfacial diffusion. The second set of equations was derived in the first part of our work, and it is based on the phase-field description of a heterogeneous binary mixture.

\subsection{Classical equations}

In the framework of the classical description of miscible liquids, the governing equations, that determine the hydrodynamics of two isothermal incompressible miscible liquids, read (see Gaponenko et al. 2006; Gaponenko \& Shevtsova 2010; Gaponenko et al. $2015 a$ )

$$
\begin{gathered}
\frac{\partial \boldsymbol{u}}{\partial t}+(\boldsymbol{u} \cdot \nabla) \boldsymbol{u}=-\nabla \Pi_{1}+\Delta \boldsymbol{u}-\frac{G s}{S c}\left(\boldsymbol{w} \cdot \boldsymbol{w}_{0}\right) \nabla C, \\
\frac{\partial C}{\partial t}+(\boldsymbol{u} \cdot \nabla) C=\frac{1}{S c} \Delta C, \\
\nabla \cdot \boldsymbol{u}=0 .
\end{gathered}
$$

These are the Navier-Stokes equation, the equation for the diffusive transport, and the continuity equation. For simplicity, dependencies of viscosity and diffusion coefficients on concentration are disregarded. These are the time-averaged equations that are written for the time-average quantities, namely, $\boldsymbol{u}$ is the time-averaged velocity, $\Pi_{1}$ is the modified pressure that is determined from an incompressibility constraint, and $C$ is the

$\dagger$ It was shown by Gaponenko et al. $(2015 b)$ that the $2 D$ model provides a good representation of the experimental observations. 
concentration that is defined as the mass fraction of one of the liquids in a mixture. It is assumed that the container that encloses the mixture is subjected to the highfrequency vibrations, with the direction given by the vector $\boldsymbol{w}_{0}=\boldsymbol{i}$, where $\boldsymbol{i}$ is a unit vector along the $x$-axis. The Navier-Stokes equation includes the vibrational force. The vector $\boldsymbol{w}$ determines the spatial variations in the pulsational velocity and it is defined by equations,

$$
\nabla \times \boldsymbol{w}=\boldsymbol{w}_{0} \times \nabla C, \nabla \cdot \boldsymbol{w}=0 .
$$

The above equations (2.1)-(2.4) are written in the non-dimensional form. The following scales were used to non-dimensionalise the equations

$$
u_{*}=\frac{\eta_{*}}{\rho_{*} L_{*}} ; \tau=\frac{L_{*}}{u_{*}} ; \Pi_{1 *}=\rho_{*} u_{*}^{2} ; w_{0 *}=a \omega ; w_{*}=\phi a \omega .
$$

Here, for the typical velocity and typical time scale, $u_{*}$ and $\tau$, we adopt the convective scales; $\eta_{*}$ and $\rho_{*}$ are the typical viscosity and density that may be taken as the average values of these quantities for pure components of a mixture; and the length scale $L_{*}$ is equal to the container's height. The scales of the pulstational fields $\boldsymbol{w}_{0}$ and $\boldsymbol{w}$ are denoted by $w_{0 *}$ and $w_{*}$. The scale of pressure field $\Pi_{1}$ is given by $\Pi_{1 *}$.

The governing equations include two non-dimensional parameters, these are the Schmidt and Gershuni† numbers,

$$
S c=\frac{\eta_{*}}{\rho D_{*}}, G s=\frac{\rho_{*} \phi^{2}(a \omega)^{2} L_{*}^{2}}{2 \eta_{*} D_{*}} .
$$

The governing equations are supplemented with the following boundary conditions,

$$
\boldsymbol{u}=0, w_{n}=0, \frac{\partial C}{\partial n}=0 .
$$

On all walls of a container, we impose the no-slip boundary condition for the average velocity, the no-penetration condition for the pulsational velocity (the equations for pulsations are inviscid, and hence one may not impose any restrictions on the tangential component of the pulsational flow), and the last boundary condition excludes the diffusive transport across the walls.

For the numerical solution of the $2 D$ problem the above equations (2.1)-(2.4) are convenient to re-write in terms of vorticity $\left(\Omega=\partial u_{x} / \partial y-\partial u_{y} / \partial x\right)$ and streamfunction $\left(u_{x}=\partial \psi / \partial y\right.$ and $\left.u_{y}=-\partial \psi / \partial x\right)$, namely,

$$
\begin{gathered}
\frac{\partial \Omega}{\partial t}+J(\Omega, \psi)=\nabla^{2} \Omega+\frac{G s}{S c} J\left(C, \frac{\partial \Phi}{\partial x}\right), \\
\frac{\partial C}{\partial t}+J(C, \psi)=\frac{1}{S c} \nabla^{2} C \\
\nabla^{2} \psi=-\Omega, \nabla^{2} \Phi=\frac{\partial C}{\partial x} .
\end{gathered}
$$

Here, $J\left(f_{1}, f_{2}\right)=\partial f_{1} / \partial x-\partial f_{2} / \partial y$, where $f_{1}$ and $f_{2}$ are two arbitrary functions; and we also introduced the velocity potential $\Phi$ for the pulsational velocity, $\boldsymbol{w}$, by equation,

$$
\boldsymbol{w}=-C \boldsymbol{w}_{0}+\nabla \cdot \Phi .
$$

The boundary conditions written with the use of the streamfunction, $\psi$, and potential,

$\dagger$ The Gershuni number is also frequently called the vibrational Rayleigh number. 
$\Phi$, read

$$
\psi=0, \frac{\partial \psi}{\partial n}=0, \frac{\partial \Phi}{\partial n}=C w_{0 n}, \frac{\partial C}{\partial n}=0 .
$$

Here, $w_{0 n}=0$ at the walls positioned at $y=0$ and $y=1$; and $w_{0 n}=1$ at two other walls that are set by the coordinates $x=0$ and $x=1$.

The numerical calculations are initiated by a state with no average motion $\left(\boldsymbol{u}_{0}=0\right)$, and with a sharp transition in the concentration field across the liquid/liquid interface,

$$
C_{0}= \begin{cases}-0.5, & \text { for } 0<y<0.5 \\ 0, & \text { at } y=0.5 \\ 0.5, & \text { for } 0.5<y<1\end{cases}
$$

\subsection{Phase-field approach}

Next, we write the governing equations for a heterogeneous binary mixture using the framework of the phase-field approach. These equations are also written in the nondimensional form, and to simplify the comparison of the numerical solutions of two different mathematical problems, the similar scales are accepted to non-dimensionalise these equations. The equations for the time-average fields read (see part 1 of this work for derivation of these equations),

$$
\begin{gathered}
\frac{\partial \boldsymbol{u}}{\partial t}+(\boldsymbol{u} \cdot \nabla) \boldsymbol{u}=-\nabla \Pi_{2}+\Delta \boldsymbol{u}-\frac{1}{M} C \nabla \mu-\frac{G s}{S c}\left(\boldsymbol{w} \cdot \boldsymbol{w}_{0}\right) \nabla C \\
\frac{\partial C}{\partial t}+(\boldsymbol{u} \cdot \nabla) C=\frac{1}{S c} \Delta \mu \\
\nabla \cdot \boldsymbol{u}=0 .
\end{gathered}
$$

The same notations as for equations (2.1)-(2.4) are used here. The Navier-Stokes equation (2.14) includes two forces in the right-hand-side, the vibrational force and the Korteweg force. The vector $\boldsymbol{w}_{0}=\boldsymbol{i}$; and the equations for the pulsation field, $\boldsymbol{w}$, are

$$
\nabla \times \boldsymbol{w}=\boldsymbol{w}_{0} \times \nabla C, \nabla \cdot \boldsymbol{w}=0 .
$$

In the phase-field approach, the diffusion flux is proportional to the gradient of the chemical potential. The equation for the chemical potential reads

$$
\mu=\frac{d f_{0}}{d C}-C n \Delta C
$$

Here $f_{0}$ is the classical part of the free energy function that sets the thermodynamic behaviour of a mixture. The shape of this function can be taken so to fit the experimental behaviour of a particular binary mixture. We use an expression that would be suitable for a wide class of binary mixtures with the upper critical (consolute) point (the components of such a mixture are miscible in all proportions when the mixture temperature is above the critical point, and the components of a mixture are partially miscible, until the equilibrium concentrations are reached, when the mixture temperature is below the critical point). In particular, the thermodynamic behaviour of such mixtures can be determined by the Landau function (see chapter XIV in Landau \& Lifshitz 2010),

$$
f_{0}=A C^{2}+C^{4} \text {. }
$$

This function was originally proposed for the description of near-critical systems, although it can be also used for the systems far from the critical point. Indeed, if $A=-\frac{1}{2}$ ( $A$ is a non-dimensional phenomenological parameter that may be either negative or 


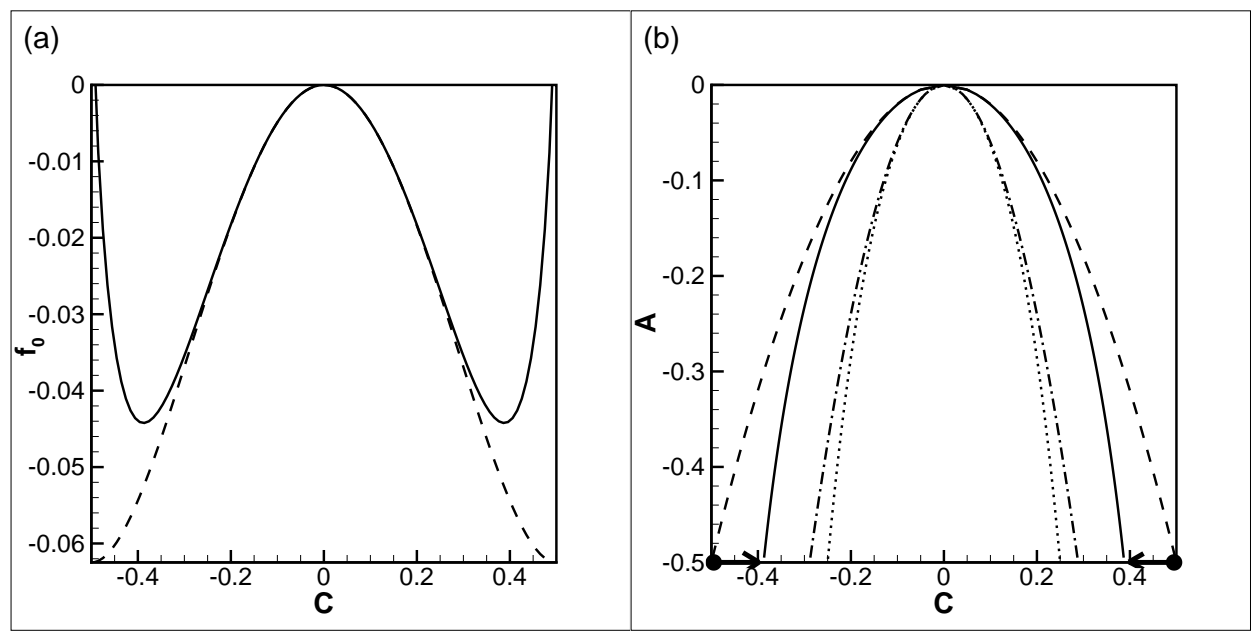

Figure 2. (a) The shapes of the free energy function (2.19), dashed line, and function (2.20), solid line, for $A=-\frac{1}{2}$. (b) The phase diagrams of a binary mixture for function (2.19), dashed line, and function (2.20), solid line; and the shapes of the spinodal curves for function (2.19), dotted line, and function (2.20), dash-dotted line. The dots indicate the initial state of the mixture, and the arrows indicate the thermodynamic transformations experienced by a mixture.

positive) function (2.19) turns into another popular expression, called a 'double-well potential', that is frequently used for numerical modelling of systems with immiscible interfaces (see e.g. Jacqmin 1999, 2000; Ding et al. 2007; Ahmadlouydarab \& Feng 2014; Prokopev et al. 2019).

In our current study, the free energy function is determined by a different expression,

$$
f_{0}=\left(A-\frac{3}{2}\right) C^{2}+\frac{3}{4}\left[\left(\frac{1}{2}+C\right) \ln \left(\frac{1}{2}+C\right)+\left(\frac{1}{2}-C\right) \ln \left(\frac{1}{2}-C\right)\right],
$$

which is also suitable for the description of mixtures with the upper critical point.

The shapes of the functions (2.19) and (2.20) are depicted in Figure 2a, and the shapes of the phase diagrams that determine the equilibrium states of a mixture are depicted in Figure $2 \mathrm{~b}$. One sees that the non-dimensional parameter $A$ sets the value of the 'mixture temperature'. The critical point is given by the 'temperature' $A=0$ and concentration $C=0$. If the mixture temperature is above the critical point, $A>0$, then the mixture is always homogeneous in equilibrium (or, liquids mix in any proportions), and if the mixture temperature is below the critical point, $A<0$, then the mixture can be either homogeneous or heterogeneous in equilibrium that is determined by the overall mass balance. One sees that two expressions (2.19) and (2.20) coincide near the critical point, and separate when $|C| \rightarrow \frac{1}{2}$. We wish to associate the values of concentration $\frac{1}{2}$ and $-\frac{1}{2}$ with two pure components of the mixture. The logarithmic terms of function $(2.20)$ restrict the range of concentration to $\left[-\frac{1}{2} ; \frac{1}{2}\right]$, while function $(2.19)$ allows overshootings (non-physical values of concentration that are either above $\frac{1}{2}$ or below $-\frac{1}{2}$ ). Function (2.20) is chosen for the current study, and the value of parameter $A$ is fixed at $-\frac{1}{2}$. Figure $2 \mathrm{~b}$ depicts the values of the initial concentrations in each phase, and the directions of the thermodynamic transformations that are experienced by a mixture. For $A=-\frac{1}{2}$, the equilibrium states of a mixture are given by \pm 0.388 . The similar thermodynamic model was earlier used for modelling the dynamics of heterogeneous mixtures with undergoing phase changes in other physical settings (see e.g. Xie \& Vorobev 2016; Vorobev \& Boghi 2016; Vorobev \& Khlebnikova 2018; Lyubimova et al. 2019). 
The governing equations (2.14)-(2.18) include the following non-dimensional parameters,

$$
\begin{gathered}
S c=\frac{\eta_{*}}{\alpha \mu_{*}}, G s=\frac{\rho_{*}^{2} \phi^{2}(a \omega)^{2} L_{*}^{2}}{2 \eta_{*} \alpha \mu_{*}}, \\
M=\frac{\eta_{*}^{2}}{\rho_{*}^{2} \mu_{*} L_{*}^{2}}, C n=\frac{\epsilon}{\mu_{*} L_{*}^{2}} .
\end{gathered}
$$

These parameters are defined by using the phenomenological constants introduced within the phase-field approach. The first two parameters are the phase-field analogues of the Schmidt and Gershuni numbers. Here, $\alpha$ is the mobility coefficient, $\mu_{*}$ is a typical value of the chemical potential, and the combination of $\alpha \mu_{*} / \rho_{*}$ plays the role of the diffusion coefficient. The phase-field equations are also characterized by two new parameters, that we call, the Mach number and the Cahn number (following Lowengrub \& Truskinovsky (1998)). The definition of the Mach number does not include the capillary constant, $\epsilon$, however, this number sets the magnitude of the Korteweg force that determines the morphology of a liquid/liquid interface. Higher Mach numbers would obviously correspond to weaker surface tension forces (making the results similar to the classical approach when the surface tension forces are disregarded), and smaller Mach numbers would correspond to the stronger interfacial stresses. The Cahn number also determines the role of the surface tension forces, as it is proportional to the capillary constant $\epsilon$. The Cahn number also sets the thickness of the transitional layer between the phases, that can be estimated as $\sqrt{-C n / A}$ (see e.g. Lowengrub \& Truskinovsky 1998). The other parameter of the phase-field approach is the 'mixture temperature' $A$.

The governing equations are supplemented with the boundary conditions. We impose the no-slip condition for the average velocity and the no-penetration condition for the pulsational flow,

$$
\boldsymbol{u}=0, w_{n}=0 .
$$

We also impose the absence of the diffusive flux at the container's walls. Since the diffusive flux is determined by the gradient of the chemical potential, this boundary condition reads,

$$
\frac{\partial \mu}{\partial n}=0
$$

Here $\boldsymbol{n}$ is a unit vector normal to the wall. In comparison with the classical equations, the phase-field equations are of the higher (forth) order in terms of the concentration field, and because of this they require one additional boundary condition that would reflect the wetting properties of the walls. In the current work, we use this boundary condition in its simplest form,

$$
\frac{\partial C}{\partial n}=0
$$

This condition assumes that the molecules of the wall interact with the molecules of the mixture components equally (in other words, the contact line is orthogonal to the interface).

The governing equations (2.14)-(2.18) written in terms of the vorticity-streamfunction read,

$$
\begin{gathered}
\frac{\partial \Omega}{\partial t}+J(\Omega, \psi)=\nabla^{2} \Omega+\frac{1}{M} J(\mu, C)+\frac{G s}{S c} J\left(C, \frac{\partial \Phi}{\partial x}\right) \\
\frac{\partial C}{\partial t}+J(C, \psi)=\frac{1}{S c} \nabla^{2} \mu
\end{gathered}
$$




$$
\begin{gathered}
\nabla^{2} \psi=-\Omega, \nabla^{2} \Phi=\frac{\partial C}{\partial x}, \\
\mu=\frac{3}{4} \ln \left(\frac{1 / 2+C}{1 / 2-C}\right)-(3-2 A) C-C n \nabla^{2} C .
\end{gathered}
$$

And the boundary conditions read

$$
\psi=0, \frac{\partial \psi}{\partial n}=0, \frac{\partial \Phi}{\partial n}=C w_{0 n}, \frac{\partial \mu}{\partial n}=\frac{\partial C}{\partial n}=0 .
$$

Finally, the initial state of the system is set by the following concentration field,

$$
C_{0}=0.495 \tanh \left(\frac{y-1 / 2}{\delta_{0}}\right) .
$$

Here, the initial interface thickness is (almost always) set by expression, $\delta_{0}=\sqrt{-C n / A}$, in exception for Figure 4 where the interfacial thickness is treated as an additional independent parameter. There is no average motion at the initial time moment $\left(\boldsymbol{u}_{0}=0\right) . \dagger$

\subsection{Numerical solution. Convergence}

The mathematical problems set for the classical and phase-field models are solved numerically in the vorticity-streamfunction formulation using the finite-difference approach. An explicit numerical scheme that uses the first-order discretization for the time derivative and the second-order discretization for the spatial derivatives is used.

To trace the intensity of the fluid motion and the intensity of the molecular mixing the following integral parameters are calculated.

(i) The total kinetic energy of the average fluid motion,

$$
E_{k}=\frac{1}{2} \int_{V} u^{2} d V
$$

where $V$ is the total volume of the fluid domain (in $2 D$, the volume is in fact replaced by an area of a fluid domain in the $x-y$ plane). This is the non-dimensional expression, and the units of energy that were used for non-dimensionalisation are $\rho_{*} u_{*}^{2} L_{*}^{2}$.

(ii) The kinetic energy of the pulsational motion,

$$
E_{w}=\frac{G s}{2 S c} \int_{V} w^{2} d V
$$

(iii) The interfacial energy,

$$
E_{i}=\frac{C n}{M} \int_{V}(\nabla C)^{2} d V .
$$

(iv) The volume of the transitional layer between the phases, that is calculated as the volume of the fluid domain with the concentration values in the range $|C| \leqslant 0.2$.

(v) The average concentration in each liquid phase. It is assumed that the position of the interface is given by the value $C=0$. Liquid 1 is determined by the negative values of the concentration field, and liquid 2 is determined by the positive values of the field of concentration. The average concentration in liquid 1 is denoted by $C_{1}$, and the average concentration in liquid 2 is denoted by $C_{2}$ (owing to the symmetry of the

$\dagger$ To simplify comparisons with other studies, we show the numerical results for the interface that is horizontal (stretches along the larger dimension of the container) in the initial moment as set in experiment (Gaponenko et al. 2015b). Under weightlessness conditions the initial shape of the interface may be different, and we also performed the simulations when the interface was curved at the initial moment, but no principal differences in the results were obtained. 


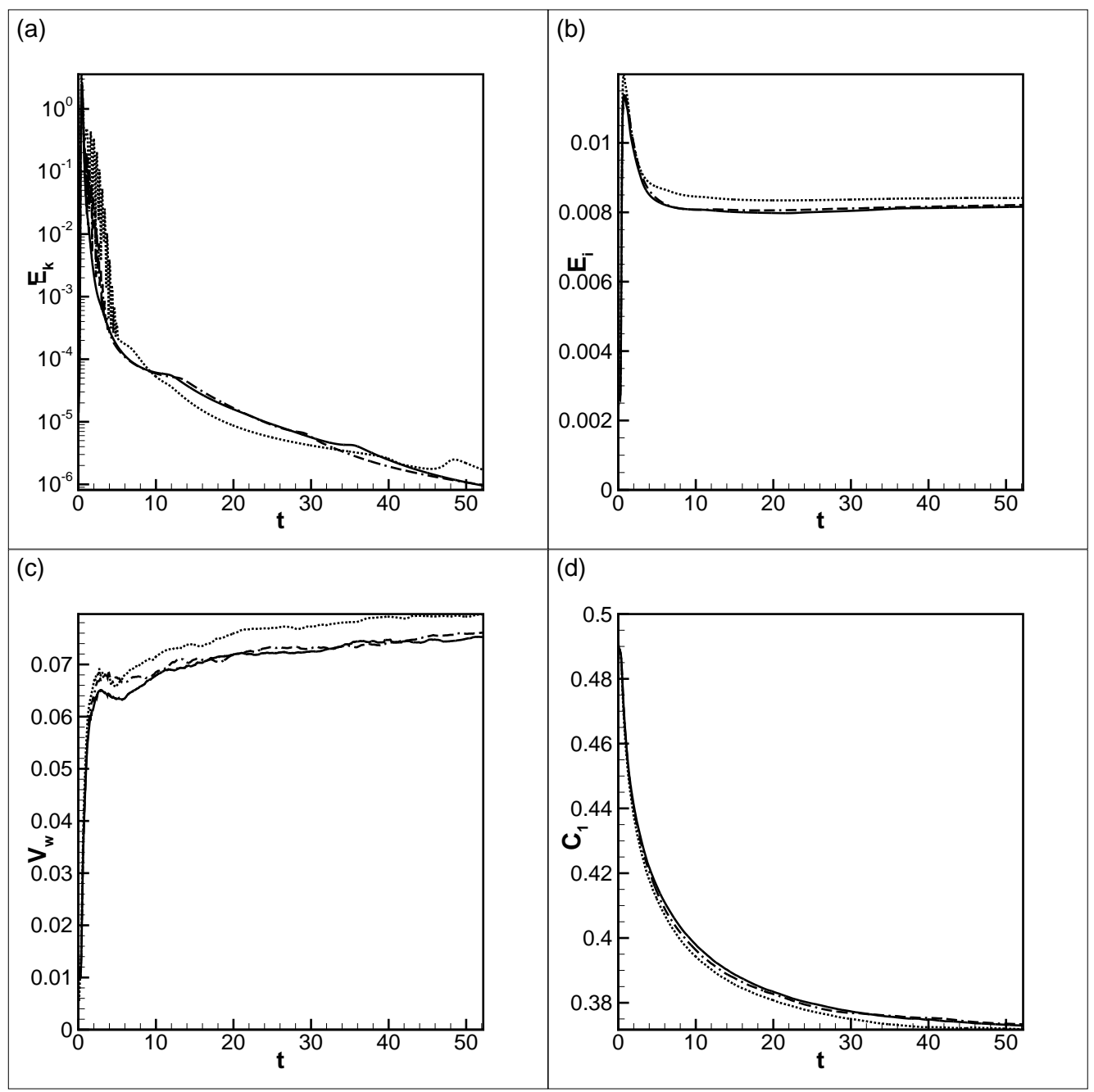

Figure 3. (a) The total kinetic energy of the average motion, (b) the interfacial energy, (c) the volume of the interface, and (d) the average concentration in one liquid vs. time. The results are shown for $A=-0.5, S c=10^{4}, G s=5 \cdot 10^{6}, M=1$, and $C n=2.5 \cdot 10^{-5}$. The dotted lines show the data obtained with $400 \times 800$ grid points, the dashed lines are for $500 \times 1000$ grid points, and the solid lines are for $600 \times 1200$ grid points, respectively.

examined problem (the symmetry of the initial state and the symmetry of the phase diagram, Figure 2b) in all calculations $C_{1}=-C_{2}$.).

The numerical convergence and the mesh-independence of the numerical simulations are illustrated by Figure 3. The required resolution is determined by the governing parameters: the better resolution is needed at greater values of the Schmidt and Gershuni numbers, which should be explained by smaller diffusive length scales and higher flow intensities, and also at smaller values of the Cahn number that correspond to smaller thicknesses of interfaces. In Figure 3, one may see that the lines that depict the time changes of different quantities clearly converge to the limiting curves.

For the phase-field results it is also important to investigate the convergence on the value of the interface thickness. The thickness of a real interface between two immiscible liquids is of the order of several molecular layers, which is equivalent to zero value for the 


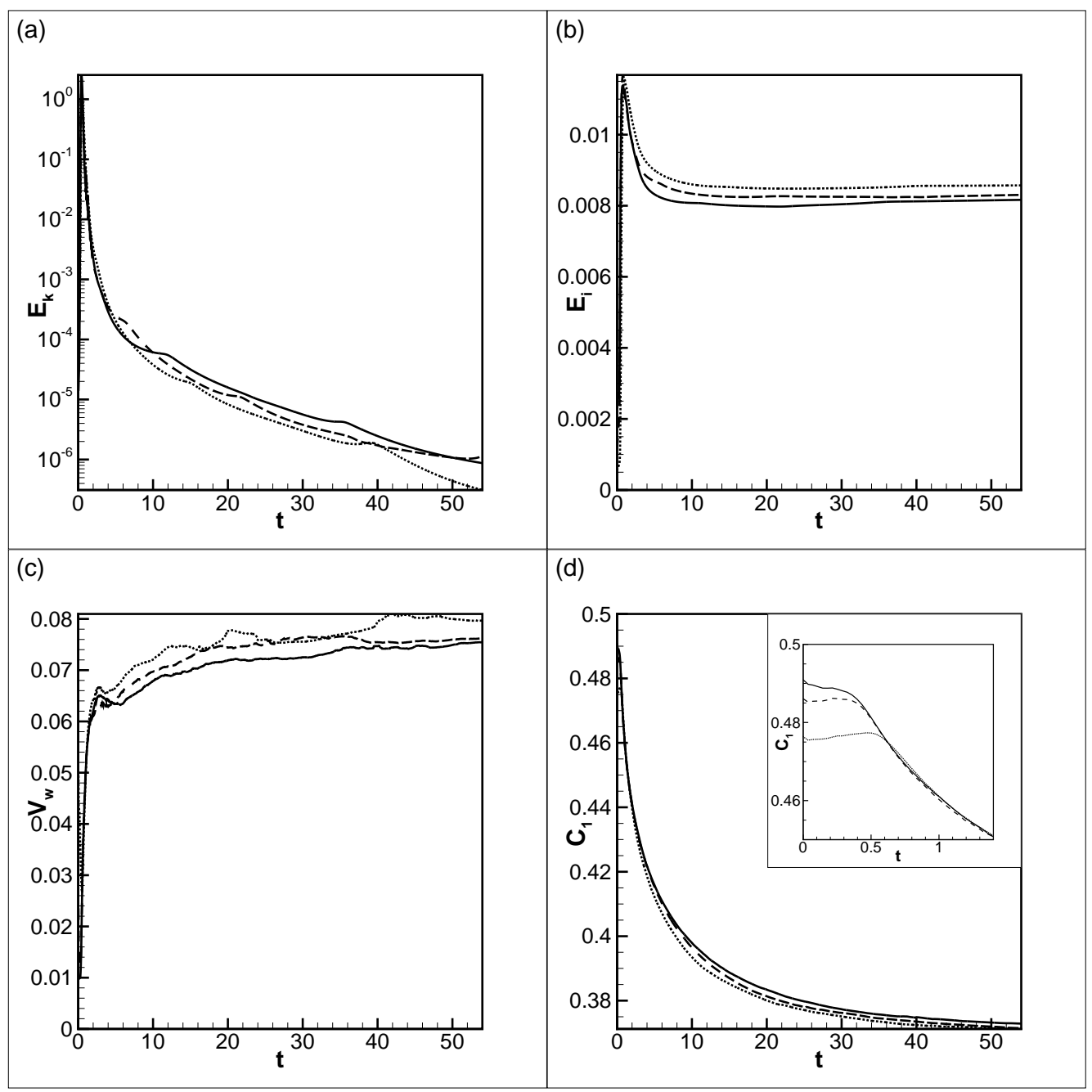

Figure 4. (a) The total kinetic energy of the average motion, (b) the interfacial energy, (c) the volume of the interface, and $(\mathrm{d})$ the average concentration in one liquid vs. time. The results are shown for $A=-0.5, S c=10^{4}, G s=5 \cdot 10^{6}, M=1$, and $C n=2.5 \cdot 10^{-5}$, and for different initial thicknesses of the interface, the solid lines shows the data for $\delta_{0}=\sqrt{-C a / A} \approx 0.007$, the dashed lines are for $\delta_{0} \approx 0.014$, and the dotted lines are for $\delta_{0} \approx 0.028$.

hydrodynamic (macroscopic) approach. The interface is represented by a transitional layer of a finite thickness for the sake of numerical simulations, and these interface thicknesses are not equal to the real thicknesses of interfaces. One however needs to show that the numerical results converge when the thickness of the interface tends to zero (tends to the real thickness). It should be mentioned that there are some cases, e.g. the interface of a near critical mixture, or the interface between two liquids miscible in all proportions after a prolonged contact, when the interfacial boundary could be quite diffusive, and in those cases both the numerical and physical thicknesses of the interfacial boundary are both finite and hence the simulations can be fulfilled with the use of the real thickness of the interface. Figure 4 depicts the data obtained for the different values of the initial thicknesses of interfaces, $\delta_{0}$. The curves may be different in the very initial moments (see e.g. the insert in Figure 4d), but they quickly converge to a single curve. 


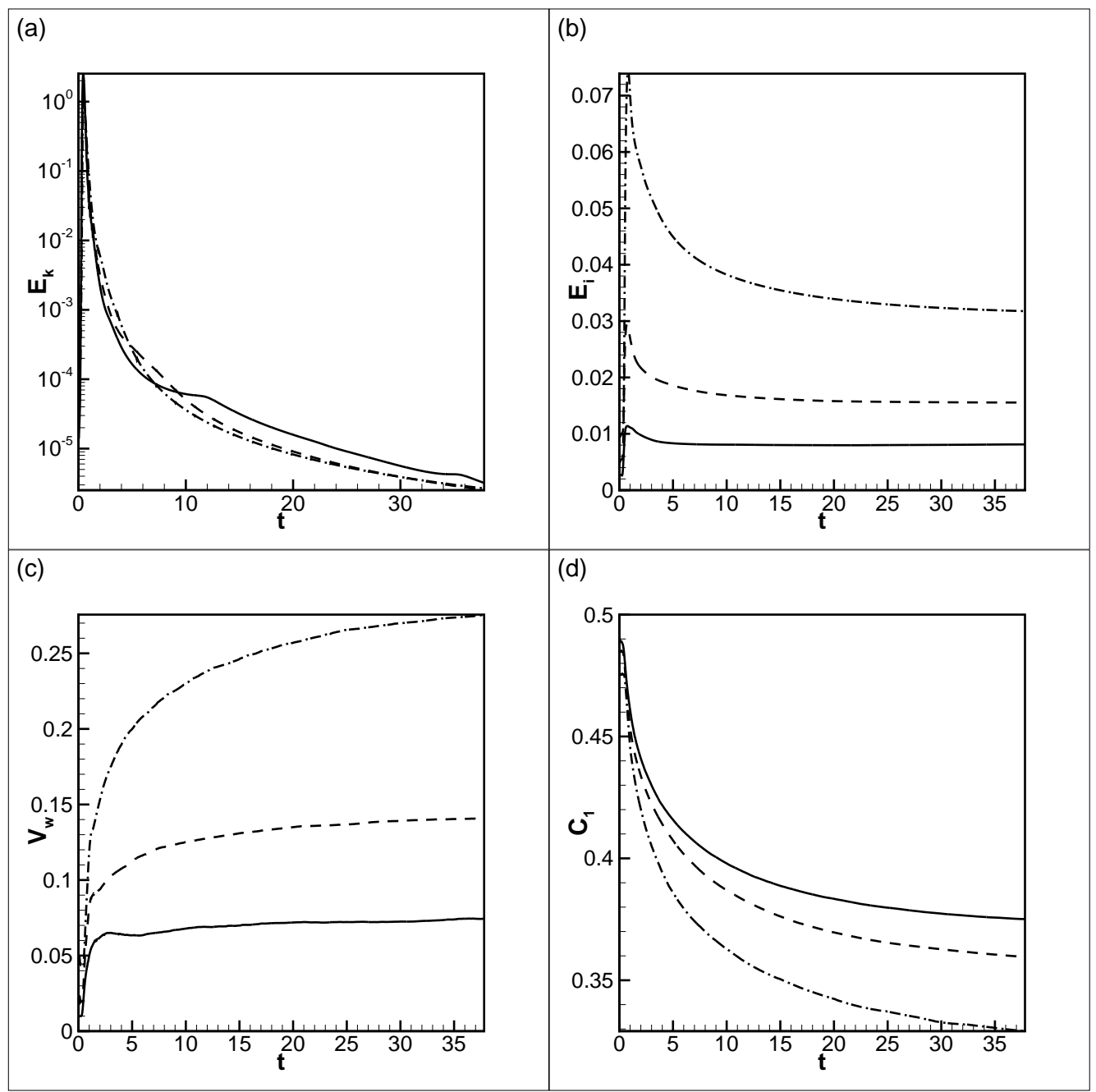

FiguRE 5. (a) The total kinetic energy of the average motion, (b) the interfacial energy, (c) the volume of the interface, and (d) the average concentration in one liquid vs. time. The results are shown for $A=-0.5, S c=10^{4}, G s=5 \cdot 10^{6}, M=1$, and $C n=2.5 \cdot 10^{-5}$ (solid lines), $C n=10^{-4}$ (dashed lines), and $C n=4 \cdot 10^{-4}$ (dash-dotted lines).

Further, the curves slowly diverge from each other, nevertheless, they stay sufficiently close to claim that our numerical results are virtually independent of the value of $\delta_{0}$.

Some of the curves in Figure 4 are plotted by taking the initial thickness of the interface different from the equilibrium value, $\sqrt{-C n / A}$. Since the initial state of a mixture differs from the state of thermodynamic equilibrium, the initial thickness of the interface (similar to the levels of concentrations in the neighbouring liquids) should in general be different from its equilibrium value. We however observe that all curves quickly converge to a single curve that is obtained when the initial interface thickness is taken equal to its equilibrium value. At this moment the whole binary mixture does not reach the state of thermodynamic equilibrium yet, and there is ongoing diffusive mass transport across the liquid/liquid boundary, although, the adjustment of the interface thickness to its equilibrium value is already completed.

In Figure 5, we investigate the numerical convergence on the interface thickness for 
the three runs that are fulfilled with the different values of the Cahn number. By altering the Cahn number we simultaneously change the interface thickness and the surface tension coefficient that is associated with the interface. The difference in the values of the interfacial energy is explained by a factor $C n$ that appears in front of expression (2.34), so the curves for the quantity, $E_{i} / C n$, would lie much closer to each other. The curves of the volume of the interfacial line also differ by a similar factor (one sees that when $C n$ is doubled the volume of the interfacial line is also doubled). The curves in Figures 5a,d demonstrate a clear convergence of the numerical results upon a gradual decrease in the values of the interfacial thickness (the distance between the curves gradually decreases when the Cahn number is taken smaller and smaller).

In conclusion, we say that the results shown in the next section of our work are obtained with the numerical grids that contain at least $450 \times 900$ grid nodes (for lower Gershuni numbers) and typically include $600 \times 1200$ grid nodes (for the runs fulfilled for higher Gershuni numbers). For the phase-field simulations, the main results are obtained for the fixed value of the Cahn number, $C n=2.5 \cdot 10^{-5}$, fixed value of parameter $A, A=-\frac{1}{2}$, and for the initial interface thickness, $\delta_{0}=\sqrt{-C n / A} \approx 0.007$.

\section{Numerical results}

\subsection{Classical approach}

We present the numerical results starting from the data obtained with the use of the classical equations (2.1)-(2.3). The similar data (for a slightly more general model that takes into account the concentration dependencies of the diffusion and viscosity coefficients) was earlier reported in the works by Gaponenko et al. (2015b).

Gaponenko et al. $(2015 b)$ presented the results in the dimensional form, and we present our data using the non-dimensional variables. The data sets can still be easily compared as all parameters that are used for our simulations were taken to be close to the experimental values. In particular, the experiment deals with the mixture of $90 \%$ water - $10 \%$ isopropanol and 50\% water - 50\% isopropanol. Owing to the dependence of the viscous and diffusion coefficients on mixture composition, it is difficult to set the single values of the Schmidt and Gershuni numbers that would work for the entire fluid domain. Depending on a particular location within the container, the Schmidt number may vary in the range of $(2030-21165)$. The range of the Gershuni numbers (for different levels of vibrations, and for different locations in the cavity) could be roughly estimated in the range of $\left(10^{4}-10^{9}\right)$ (the upper end of this range corresponds to rather large amplitudes of vibrations that are even greater than the size of the container). Gaponenko et al. $(2015 b)$ avoided this problem by solving the equations in the dimensional form and by taking into account the density dependencies of the phenomenological parameters. We neglect the concentration dependencies of the viscosity and diffusivityt, and this allows us to characterise the mixture by a single Schmidt number (which we set $S c=10^{4}$ for all calculations) and by a single Gershuni number (for a fixed level of the vibrational forcing). Finally, we mention that the real convective time scale varies in a range of $(16.6-39.08) \mathrm{s}$. The convective time scale is used as a unit of the non-dimensional time in our work, and thus, we may roughly say that one unit of the non-dimensional time is approximately 30 s.

The data shown in Figure 6 and 7 present typical evolutions of an interface in a

$\dagger$ In the phase-field theory, that is considered later, the concentration dependence of the diffusion coefficient is given by free energy function (2.20), namely $\sim d^{2} f_{0} / d C^{2}$, however, in the classical approach the diffusion coefficient is assumed to be just constant. 


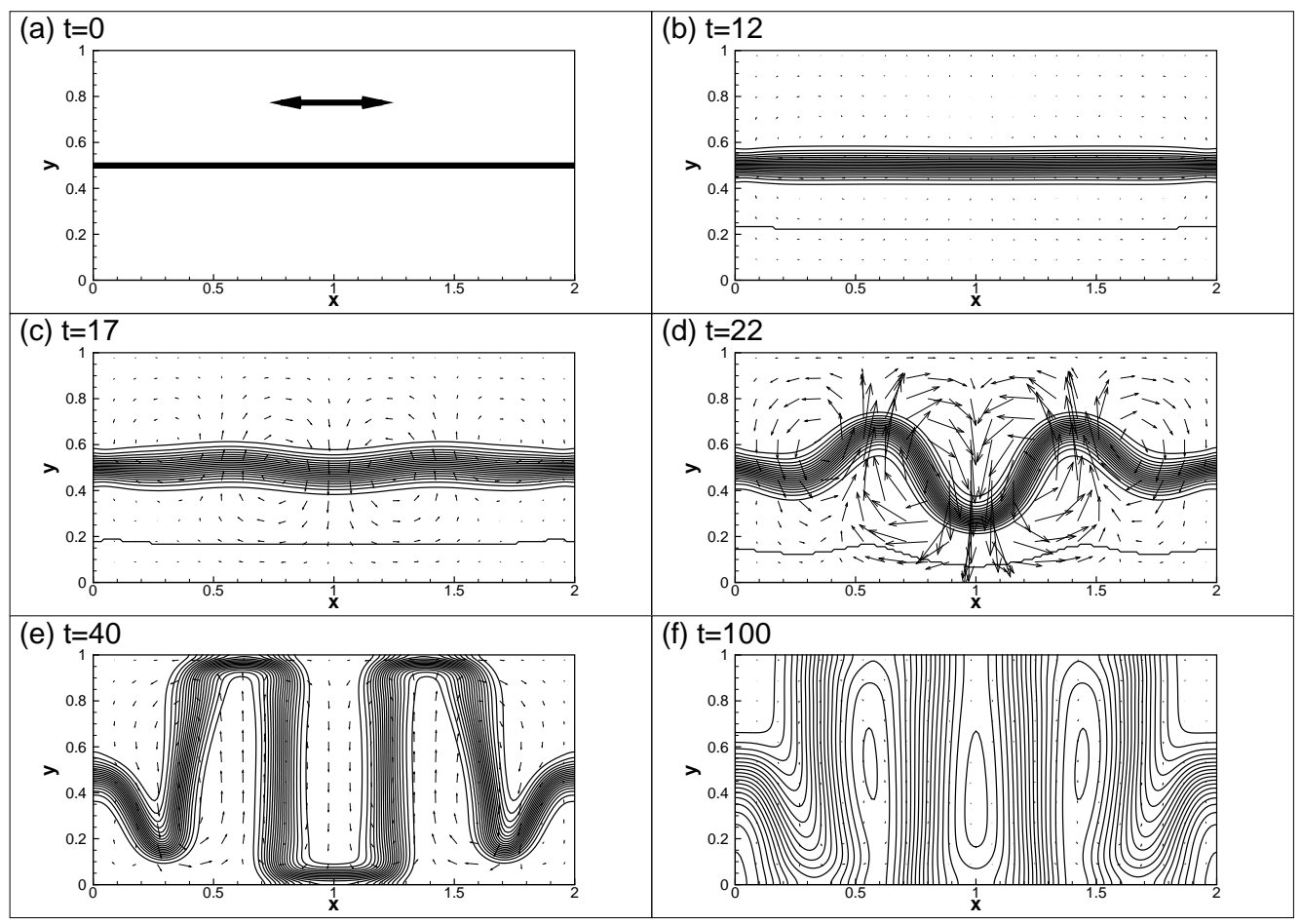

FiguRE 6. The snapshots of the fields of concentration (isolines) and velocity (vectors). The data are obtained for the run fulfilled with the use of the classical model for $S c=10^{4}$ and $G s=10^{5}$. The double-arrow line in (a) shows the direction of vibrations.

binary system that is subjected to high-frequency vibrations of different intensities. The snapshots shown in Figure 6 demonstrate a good general agreement of the numerical results with the experimental observations reported by Gaponenko et al. $(2015 b)$. In the beginning, the vibrations induce some weak average flows in both fluid domains. The magnitude of the induced flow grows in time, but when the vibrational forcing is weak (lower Gershuni numbers), during the numerical run the induced flows remain too weak to disturb the interface shape: the interface remains flat and it just slowly smears in time. If however the vibrational forcing is stronger, the average motion induced by the vibrations destabilizes the flat interface, eventually forming a pillar-shaped structure in a container. The vertical growth of the pillars is restricted by the container's height, and when the pillars reach the upper and lower walls of the cavity (occupy the full height of the cavity), the intensity of the average and pulsation motions decay. The further development of the pillars occurs much slower, and it is characterised by slow diffusive smearing of immovable pillar's walls. These pillar-shaped structures represent a phenomenon of a frozen relief (or frozen waves).

In Figure 6f, that corresponds to the time moment of 100 (about an hour in physical time), the interface is very diffuse. The experimental results (see Gaponenko et al. 2015a,b; Shevtsova et al. 2015, 2016) do not include the pictures with so diffusive interfacial boundaries, which may be explained by a difficulty of processing similar images or by time restrictions of parabolic flights that were used to achieve the weightlessness conditions. It is also interesting to note that a typical diffusive time scale is of the order of $S c$, and hence one expects a noticeable diffusive smearing over the time period of the order of $10^{4}$. Figure 6 f clearly shows that the diffusive smearing is substantially sped up 


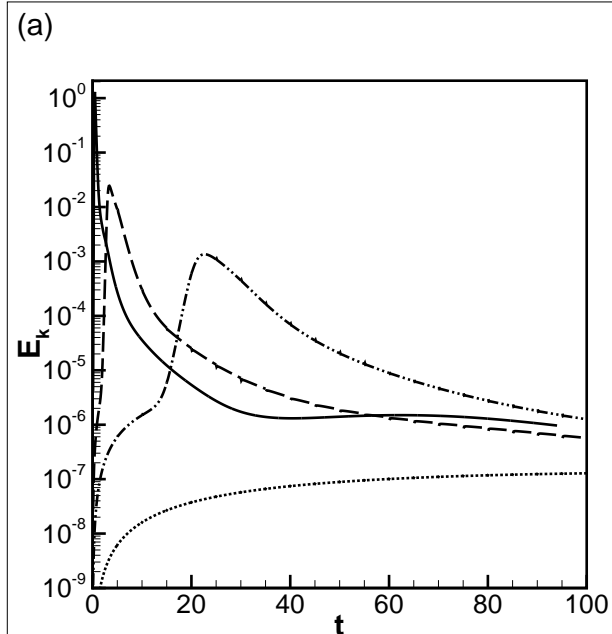

(c)

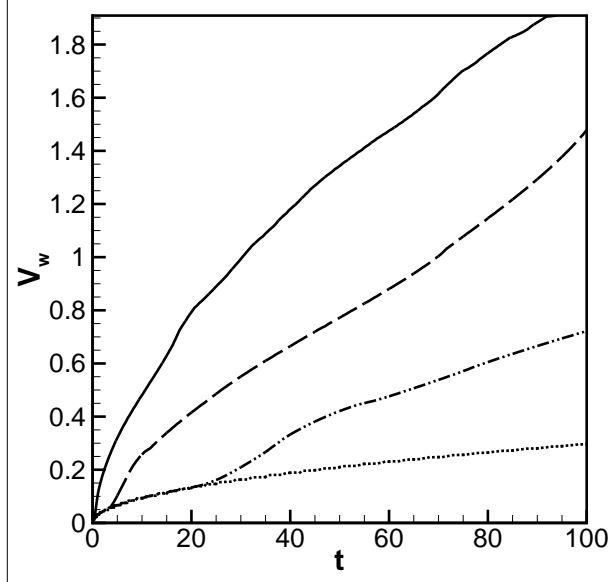

(b)

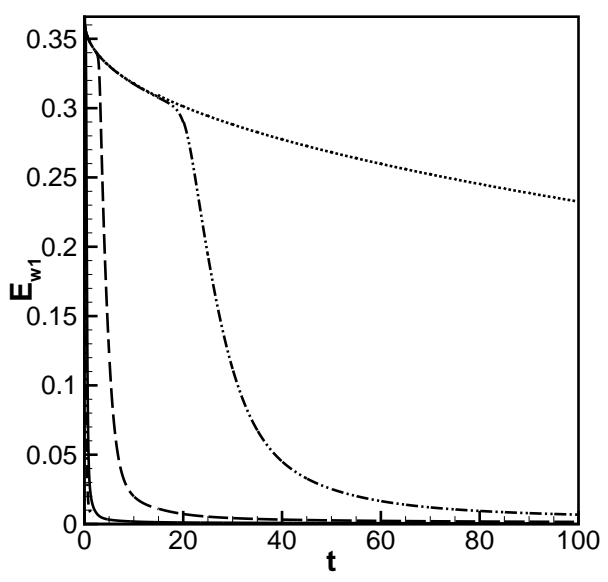

(d)

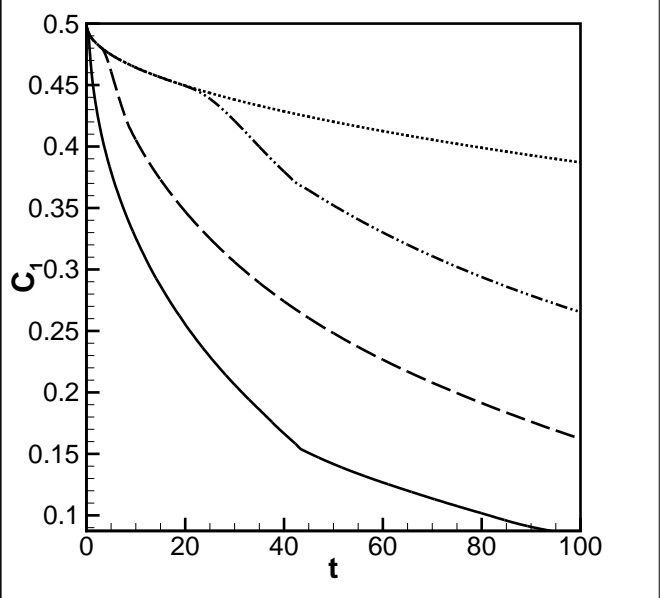

Figure 7. (a) The kinetic energy of the average flow, (b) the kinetic energy of the pulsation flow (here $E_{w 1} \equiv E_{w} S c / G s$ ), (c) the volume of the interface, and (d) the average concentration in liquid 1 vs. time. The data are obtained for the run fulfilled with the use of the classical model for $S c=10^{4}$ and $G s=10^{4}$ (dotted line), Gs $=10^{5}$ (dash-dot-dotted line), Gs $=5 \cdot 10^{5}$ (dashed line), and $G s=5 \cdot 10^{6}$ (solid line).

by the vibrational forcing, so the liquids become nearly indistinguishable after the time period of 100 , even under the action of relatively moderate vibrations $\left(G s=10^{5}\right)$. Figure 6 f also answers the Wolf's question (Wolf 2018) on a long-term stability of the frozen waves: one sees that the frozen waves formed on a miscible interface remain stable at least until the interfacial boundary can be visually identified.

Figure $7 \mathrm{a}$ shows the time changes of the total kinetic energy of the average flow for the different Gershuni numbers. At $G s=10^{4}$ the intensity of the induced average flow grows with time, however, at least for the duration of the numerical run the flow always remains too weak to affect the interface shape. At $G s \geqslant 4 \cdot 10^{4}$, at some moment the slower growth of the kinetic energy is suddenly replaced by a faster growth. This happens at the moment of the interface reconstruction that eventually results in the formation of several 'frozen' pillars stretched from the bottom to the top wall of the container. When 


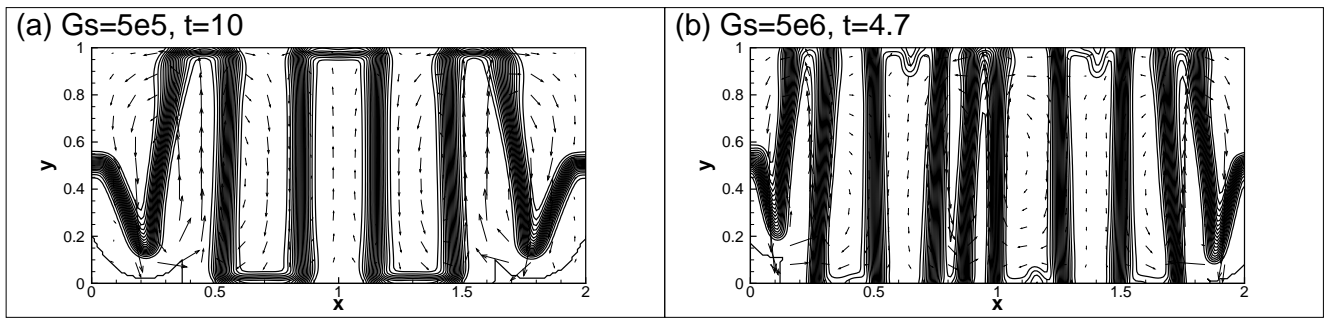

Figure 8 . The shape of an interface subjected to the vibrations with $G s=5 \cdot 10^{5}$ (a) and $G s=5 \cdot 10^{6}$ (b). The data are obtained for the classical model, for $S c=10^{4}$.

the pillars are fully set the intensity of the fluid motion drops to almost zero. Figure 7b depicts the time changes of the kinetic energy of the pulsation flow. The pulsation flow has the highest intensity in the beginning of the numerical run when the liquid/liquid interface is sharp, and the initial diffusive smearing of an interface slowly decreases the pulsation motion. At the moment of the interface reconstruction, the intensity of the pulsation flow rapidly decays. Figure $7 \mathrm{c}$ shows how the volume of the interfacial zone grows with time. Again, the rapid increase of the volume corresponds to the interface reconstruction. Figure $7 d$ shows the time changes of the average concentrations in one of the liquids. The reconstruction of the interface increases the molecular mixing that is to be explained by the growth of the interface length (i.e. the contact area of two liquids).

In Figure 7 one notices that while the interface is flat the dynamics of the binary mixture is solely determined by the diffusive process. In the later moments, when the frozen pillars are fully set, the hydrodynamic motion in the container is also absent, and the evolution of a mixture is again determined by diffusion. Nevertheless, there are clear differences between the initial and later dynamics of the mixture: the initial dynamics is independent of vibrations, and the later dynamics occurs differently for the vibrations of different intensities. This needs to be explained by the dependence of the shape of the frozen relief (the different number of the pillars in the container) on the Gershuni number. The greater the level of vibrations is, the more pillars are formed in the container, as seen in Figure 8. The liquid/liquid contact surface is obviously greater when there are more pillars, which explains the differences in the rates of molecular diffusion at the later time periods.

In Figure 7 one sees that the reconstruction of an interface starts earlier, occurs faster, and the maximum values of the kinetic energy are greater for the runs with higher Gershuni numbers, which is also illustrated in Figures 9a,b. In Figure 9c,d the horizontal size of the pillars characterized by the wave-length that is calculated by taking the ratio of the numbers of several fully-formed pillars (two pillars form a 'wave') in a central part of the container over the width of the region occupied by these pillars.

A particular attention of the current work is addressed to the question of whether the formation of the frozen waves requires the vibrational forcing with an intensity over a certain threshold level. In the classical theory (2.1)-(2.3), the surface tension is completely disregarded. In accordance with equation (1.1) under weightlessness conditions the appearance of the frozen waves may happen in the presence of high-frequency vibrations of any intensity, which could be easily understood from the analysis of equations (2.1)-(2.3) where the vibrational force is the only term capable of setting the interfacial shape. In our calculations (for $S c=10^{4}$ ) the minimum Gershuni number that allows the formation of the frozen relief is $G s_{c}=4 \cdot 10^{4}$, and as one sees in Figure 7 the interface remains flat for $G s=10^{4}$ for the duration of the numerical run, i.e. for $t<100$.

The simulations fulfilled for the different levels of the Schmidt number reveal a strong 


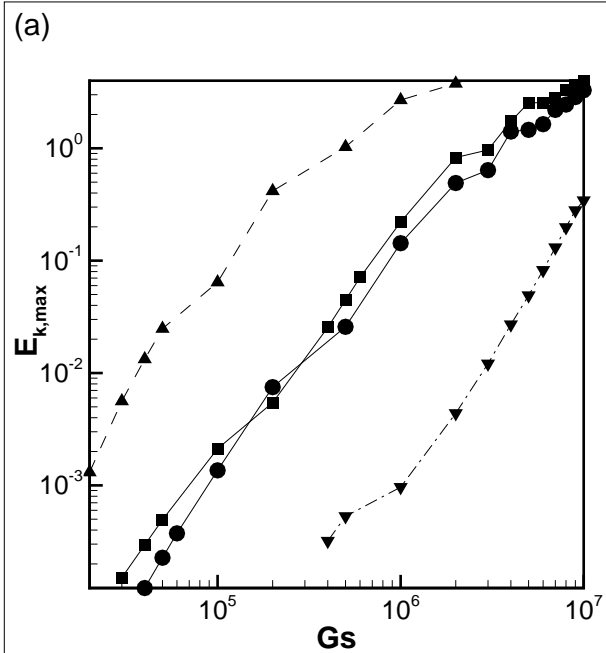

(c)

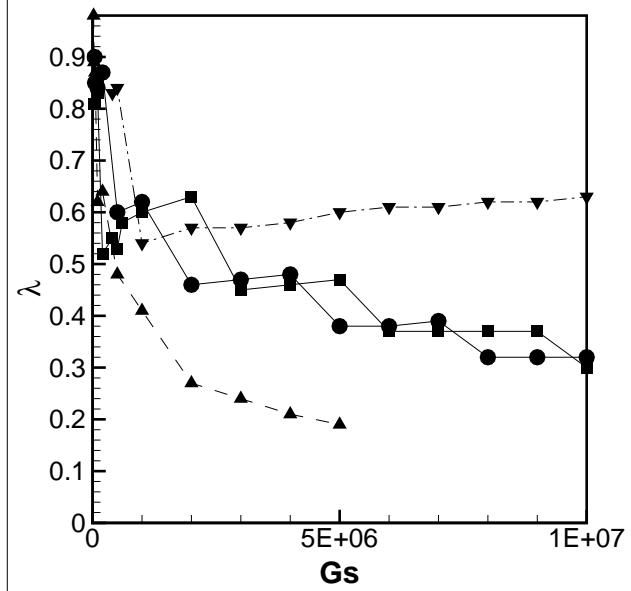

(b)

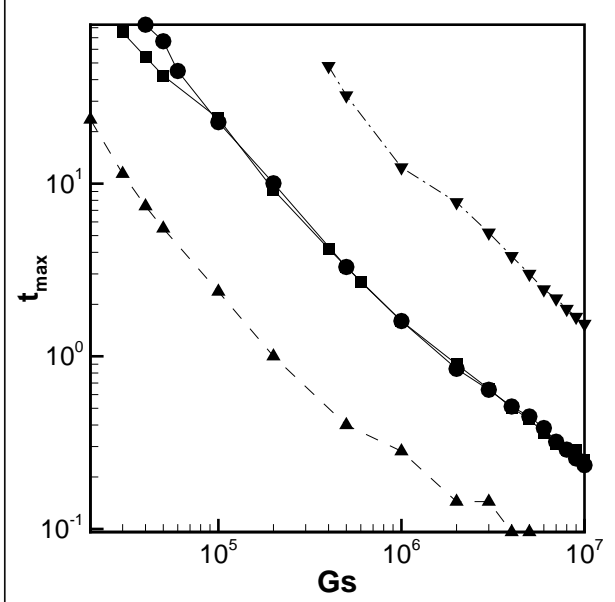

(d)

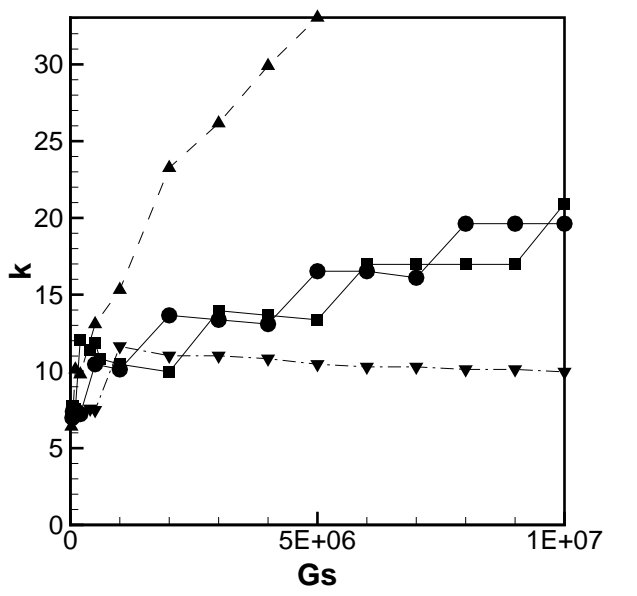

Figure 9. (a,b) The maximum value of the kinetic energy of the average flow during the evolution of a binary mixture and the time moments when the kinetic energy is maximum vs. the Gershuni number. (c,d) The wavelengths and the wavenumbers that characterise the periodicity of the frozen pillars in the horizontal dimension vs. the Gershuni number. The data are obtained for the classical model ('circles' for $S c=10^{4}$, 'triangles' for $S c=10^{3}$, and 'nablas' for $S c=10^{5}$ ) and for the phase-field simulations ('squares' for $S c=10^{4}, M=1, C n=2.5 \cdot 10^{-5}$, and $A=-0.5)$.

influence on this parameters, which however can be easily explained by the fact that the amplitude of the vibrational force in equation (2.1) is set by the ratio Gs/Sc. Figure 10 shows the dependencies of the maximum value of the kinetic energy during the reconstruction of the interface and the time moment when the kinetic energy reaches the maximum value as functions of the ratio $G s / S c$. In the new coordinates the curves obtained for different Schmidt numbers almost coincide when $(G s / S c) \rightarrow 0$. In addition, one sees that when $(G s / S c) \rightarrow 0$ the time required for the formation of the waves increases exponentially. When the time needed for the reconstruction of an interface becomes too long, the interface gets too diffusive (practically indistinguishable) before hydrodynamic motion has time to reshape the interface. Moreover, the interface smearing 


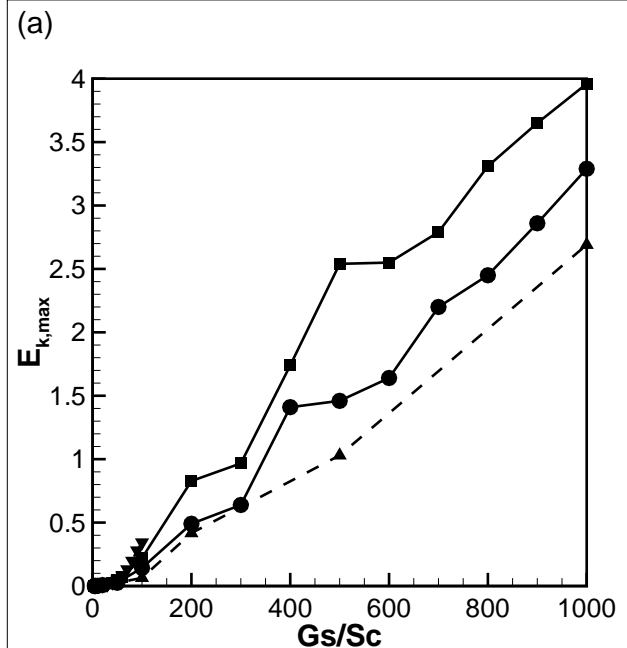

(c)

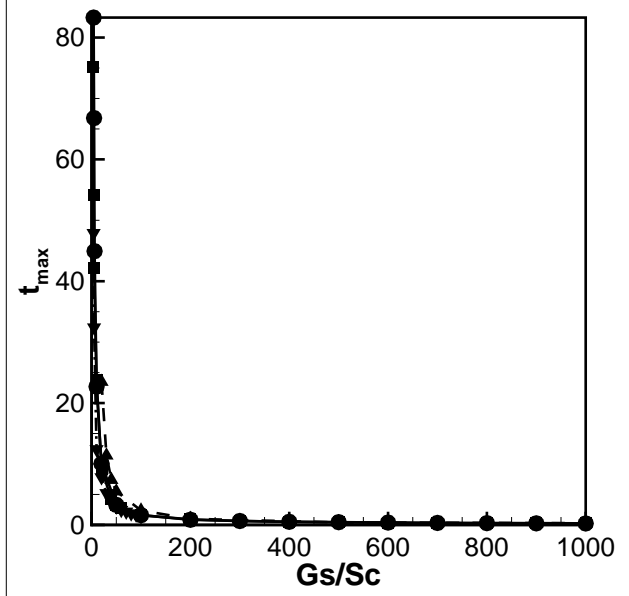

(b)

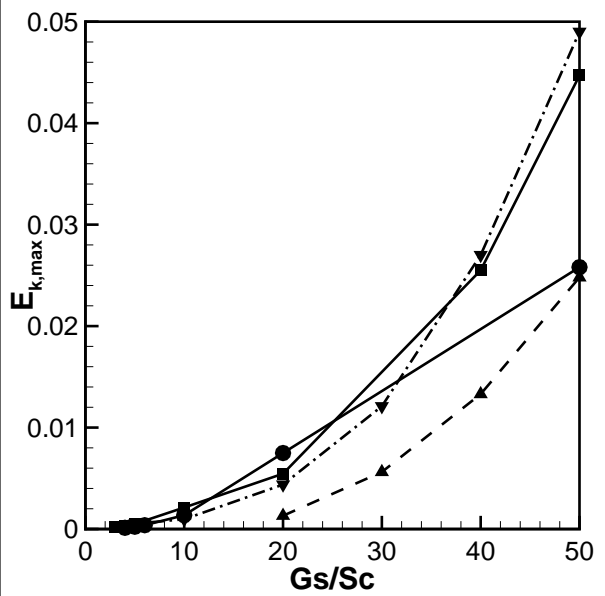

(d)

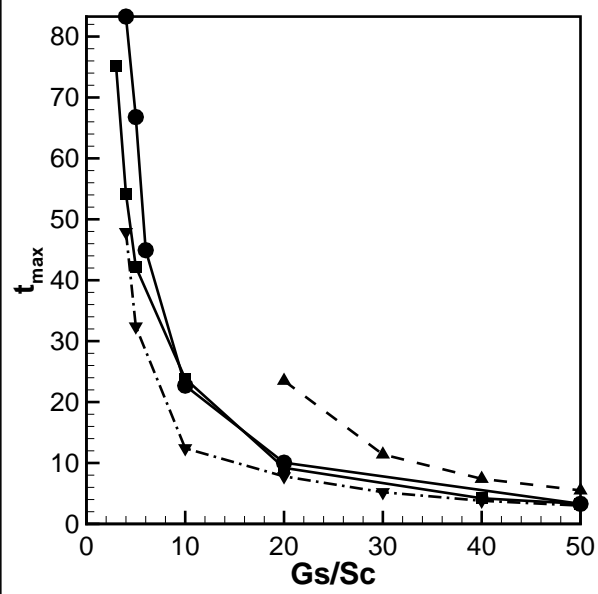

Figure 10. The maximum value of the kinetic energy of the average flow during the evolution of a binary mixture (a,b) and the time moments when the kinetic energy is maximum (c,d) vs. $G s / S c$ (the ratio of Gershuni and Schmidt numbers). The data are obtained for the classical model ('circles' for $S c=10^{4}$, 'triangles' for $S c=10^{3}$, and 'nablas' for $S c=10^{5}$ ) and for the phase-field simulations ('squares' for $S c=10^{4}, M=1, C n=2.5 \cdot 10^{-5}$, and $A=-0.5$.

reduces the magnitude of the vibrational forcing even further, making its influence even less significant over the time. In this case the isolines of the concentration field become only slightly distorted by the hydrodynamic motion, so that the formation of the frozen waves cannot be seen. We observe such a behavior for $(G s / S c) \leqslant 4$.

In Figure 9c one sees that the longest structures that are formed in the container have the wavelength of about 0.85 (for $G s=5 \cdot 10^{5}$ and $S c=10^{4}$ ), which corresponds to three pillars (see Figure 6e). When the Gershuni number is increased the number of the pillars that are formed in the container also grows. Although, as can be seen from Figures $9 \mathrm{c}, \mathrm{d}$, the growth in the number of the pillars does not happen smoothly: there is a sequence of the critical Gershuni numbers when two new pillars (one wave) are added that also makes the other pillars thinner so the wavelength jumps to a smaller value. In particular, for $S c=10^{4}$, the changes of the pattern of the frozen waves occur at 


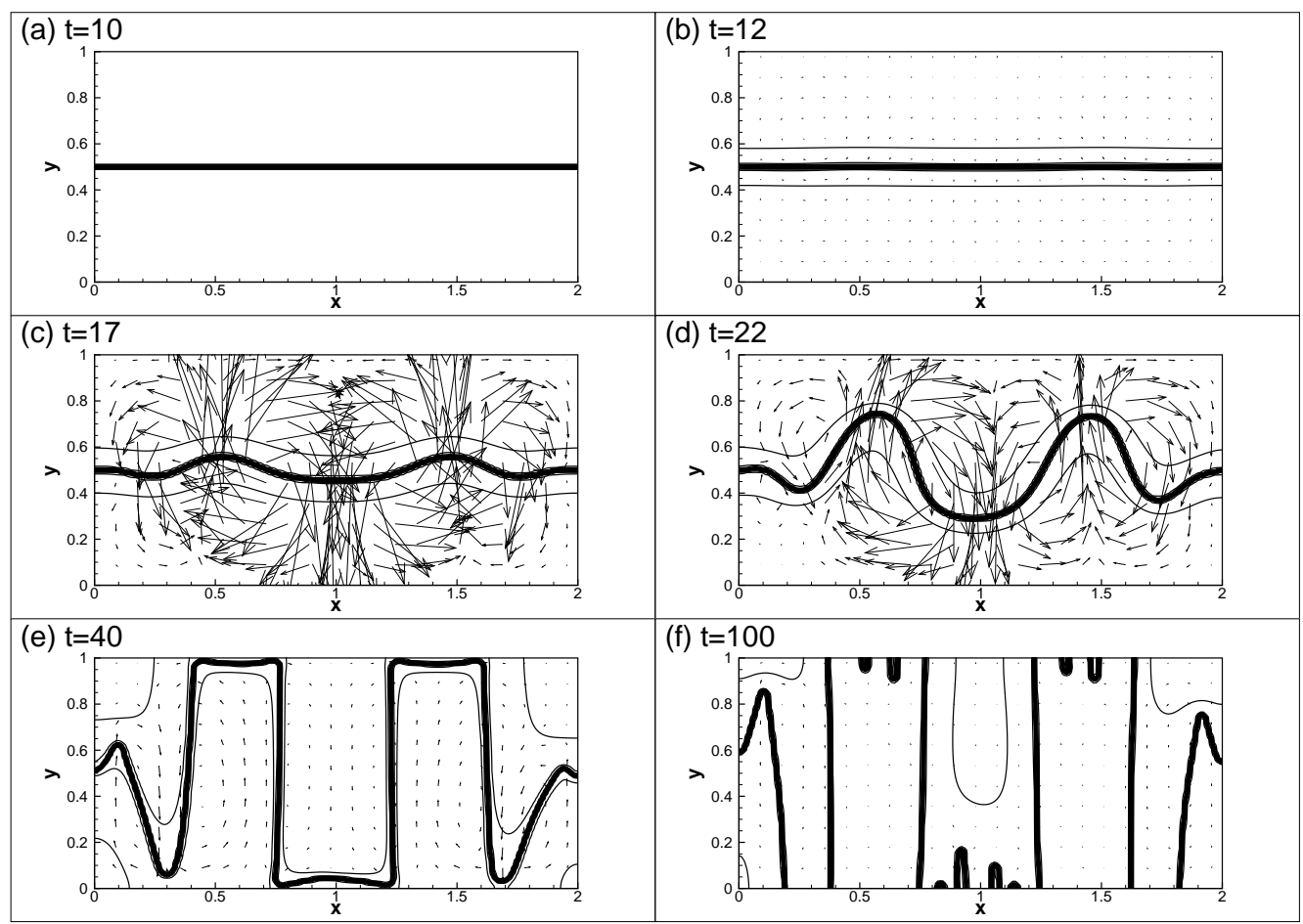

FiguRE 11. The snapshots of the fields of concentration (isolines) and velocity (vectors). The data are obtained with the use of the phase-field model for $S c=10^{4}$ and $G s=10^{5}, M=1$, $A=-0.5$, and $C n=2.5 \cdot 10^{-5}$.

approximately $G s=5 \cdot 10^{5}$ (five pillars), Gs $=2 \cdot 10^{6}$ (seven pillars), Gs $=5 \cdot 10^{6}$ (nine pillars), $G s=8 \cdot 10^{6}$ (eleven pillars), and so on. At larger Gershuni numbers the pillars become irregular (non-symmetrical), as can be seen in e.g. Figure $8 \mathrm{~b}$.

\subsection{Phase-field approach}

Figures 11 and 12 illustrate the development of the frozen waves in a heterogeneous binary mixture which evolution is modelled using the phase-field approach. Figure 11 shows the fields of concentration and velocity and Figure 12 shows the field of chemical potential. To facilitate the comparisons of the phase-field and classical results the phase-field calculations are performed for the same values of the Schmidt and Gershuni numbers. The phase-field approach, however, also requires the values of three additional parameters: the Mach number, that controls the magnitude of the Korteweg force, is initially set to an intermediate value of 1 ; the 'mixture temperature' is set so to allow some diffusion between the phases, $A=-0.5$, and the Cahn number is $C n=2.5 \cdot 10^{-5}$.

Within the phase-field approach the rate of diffusion is determined by the field of chemical potential. At the initial moment the chemical potential has a sharp transition across the interface (see Figure 12a). During the evolution the magnitude of the gradient of the chemical potential rapidly decreases, reducing the intensity of interfacial diffusion, so the diffusive transport becomes completely non-existent at the last snapshot when the binary system almost reaches its state of thermodynamic equilibrium. For $A=-0.5$, the equilibrium concentrations in each phase are \pm 0.388 (if the capillary and vibrational 


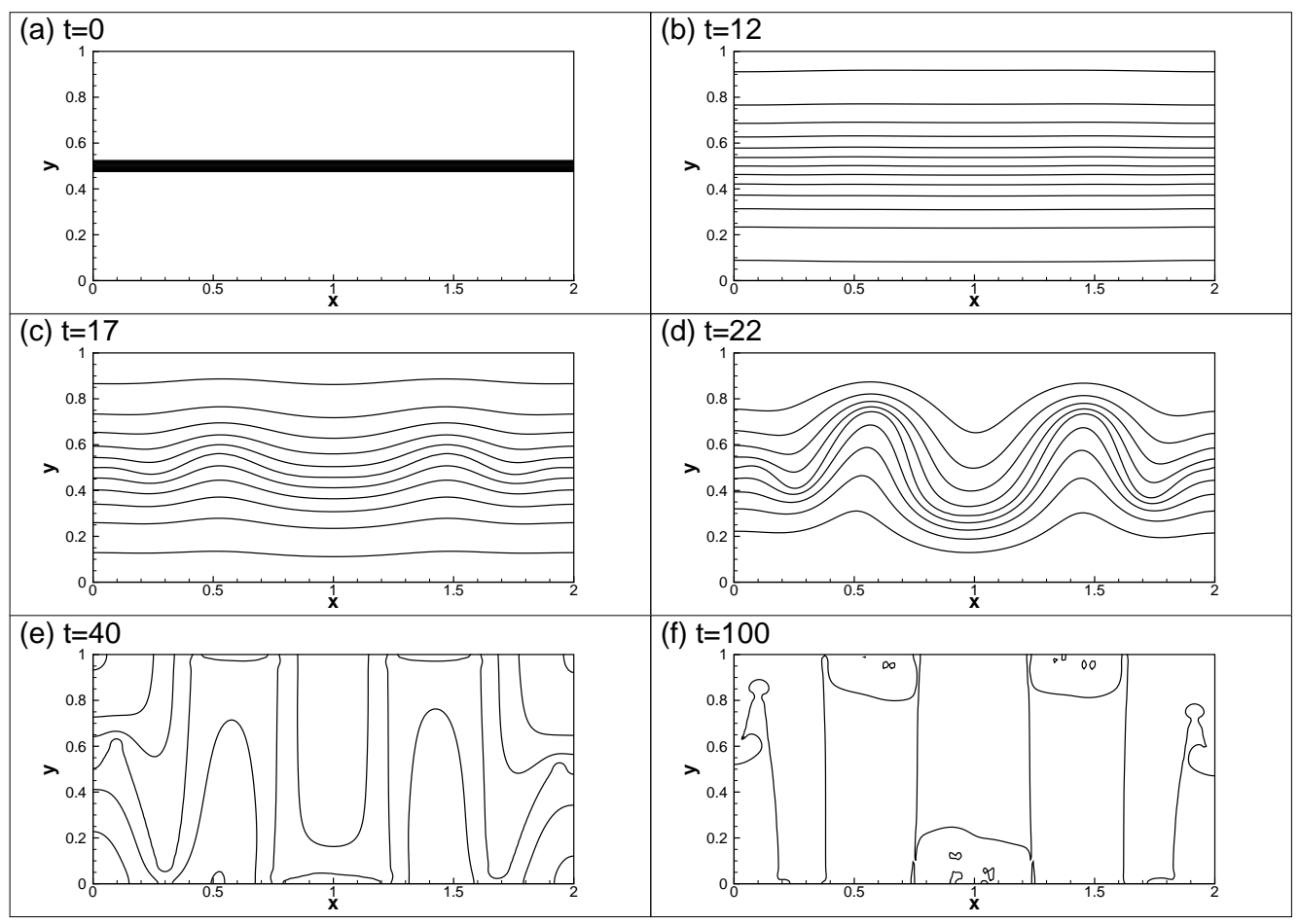

Figure 12. The snapshots showing the isolines of the of the field of chemical potential. The data are obtained with the use of the phase-field model for $S c=10^{4}$ and $G s=10^{5}, M=1$, $A=-0.5$, and $C n=2.5 \cdot 10^{-5}$. The same levels of the chemical potential are depicted in all pictures.

effects are totally neglected), and in Figure $13 \mathrm{~d}$ the average concentration $C_{1}$ is quite close to this value near the end of the numerical run $(t \sim 100)$.

The snapshots for the classical and phase-field theories look very similar, which indicates that at $M=1$ the Korteweg force remains too weak to significantly affect the dynamics of the mixture. The visible difference between Figures 11 and 6 is the level of interface smearing: in the case of the classical approach the thickness of the interface constantly grows, while in the phase-field simulations the interface thickness remains constant almost during the entire numerical run. Another difference between the phasefield and classical results is that for lower Gershuni numbers, when the interface remains flat $\left(G s=10^{4}\right.$ in Figures $7 \mathrm{a}$ and $\left.13 \mathrm{a}\right)$, the intensity of the average flow keeps growing in the classical case, and the level of energy starts decaying at some moment in the case of the phase-field simulations. The formation of the frozen waves occurs due to development of the average motion in the neighboring liquids, and when this motion becomes critically strong, the shape of the interface is changed. The latter observation indicates that for $M=1$ the threshold Gershuni number is $G s_{c}=3 \cdot 10^{4}$.

Similarities between the classical and phase-field results are also underlined in Figures 9 and 10, where the phase-field data set is distinguished by square symbols. All general features of the dynamics of the binary mixture are very similar to the classical results: the maximum values of the kinetic energy, the time moments when the kinetic energy has a peak, and the periodicity of the formed waves almost coincide. Some differences however appear at the higher Gershuni numbers: the development of the instability is characterised by more intensive flows, and the formation of additional waves in a container occurs at 

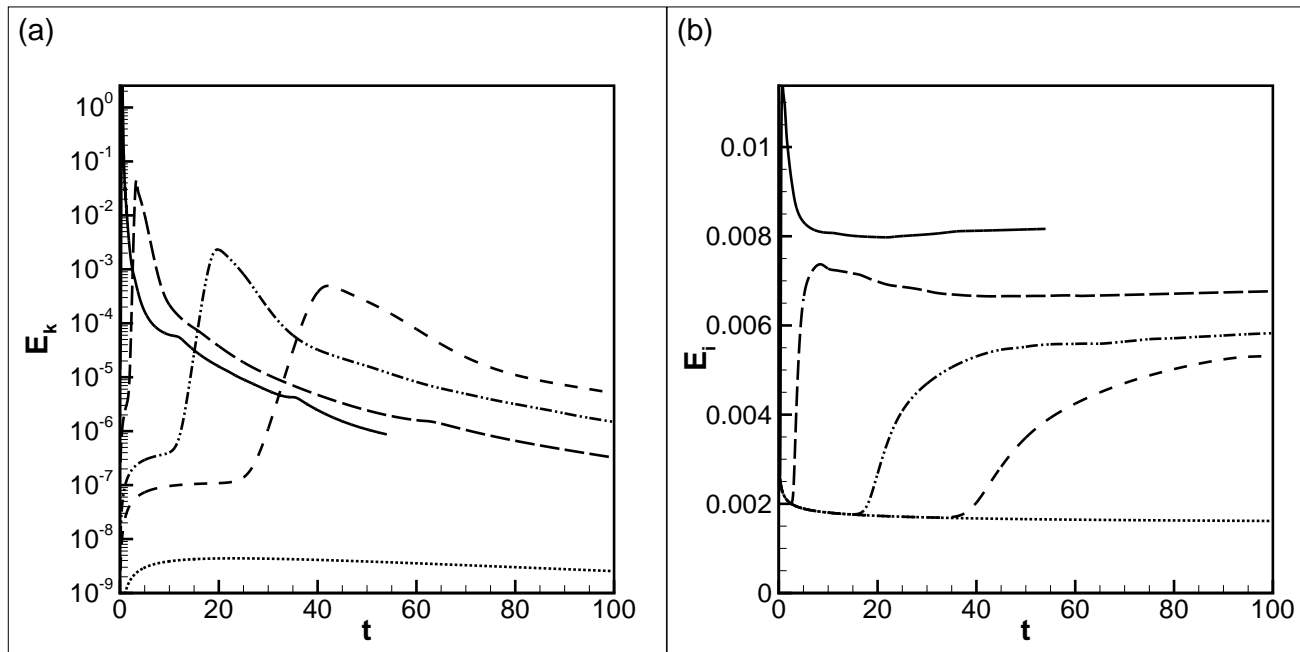

(c)

(d)
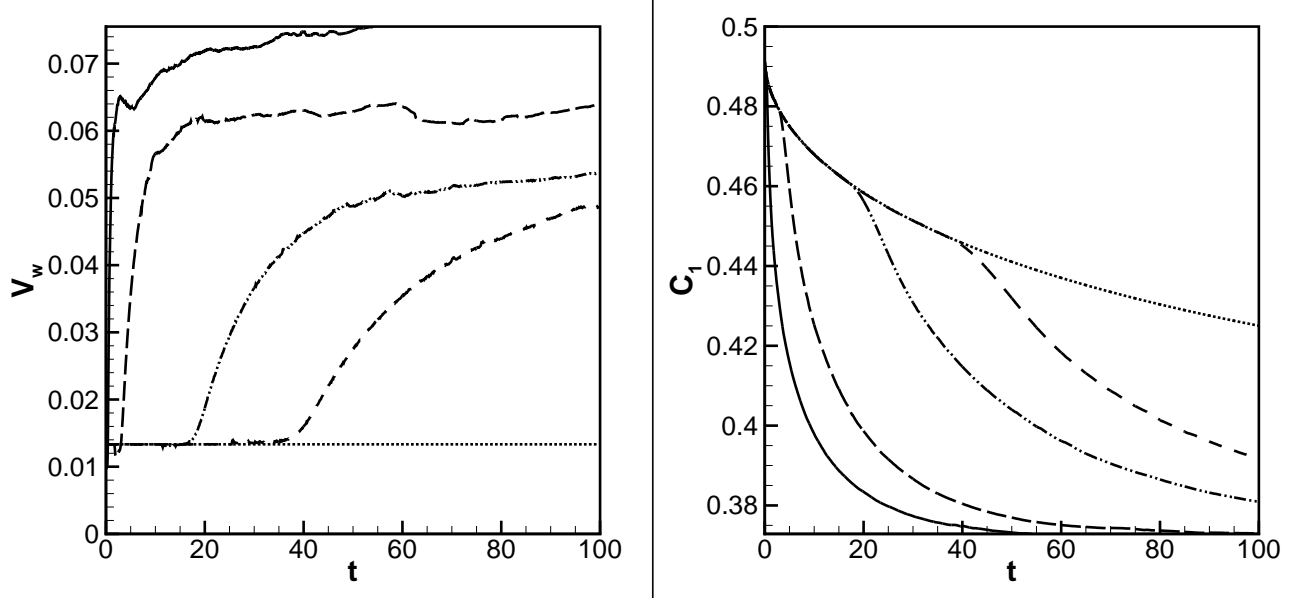

Figure 13. (a) The kinetic energy of the average flow, (b) the interfacial energy, (c) the volume of the interface, and (d) the average concentration in liquid 1 vs. time. The data are obtained for the runs fulfilled with the use of the phase-field model for $S c=10^{4}, M=1$, $A=-0.5, C n=2.5 \cdot 10^{-5}$, and $G s=10^{4}$ (dotted line), Gs $=5 \cdot 10^{4}$ (dashed line), Gs $=10^{5}$ (dash-dot-dotted line), $G s=5 \cdot 10^{5}$ (long-dashed line), and $G s=5 \cdot 10^{6}$ (solid line).

slightly higher Gershuni numbers as compared with the classical simulations, which is to be explained by the action of the surface tension forces. Namely, three pillars are formed at $G s>5 \cdot 10^{4}$, five at $G s>2 \cdot 10^{5}$, seven at $G s>3 \cdot 10^{6}$, nine at $G s>6 \cdot 10^{6}$, and eleven at $G s>10^{7}$. The clear differences are also seen at very low Gershuni numbers, closer to the instability threshold. Here, the dynamics of a mixture that is described by a classical model is characterised by stronger interface smearing, which reduces the magnitude of the vibrational force and slows down the development of the frozen wave instability, as compared to the mixture that is described using the phase-field approach.

The above results show that the initially chosen value of the Mach number, $M=$ 1 , is too low to generate substantial differences between the classical and phase-field simulations. To reveal stronger differences we perform the set of numerical simulations for smaller Mach numbers, $M<1$. We however found that the influence of the Korteweg 


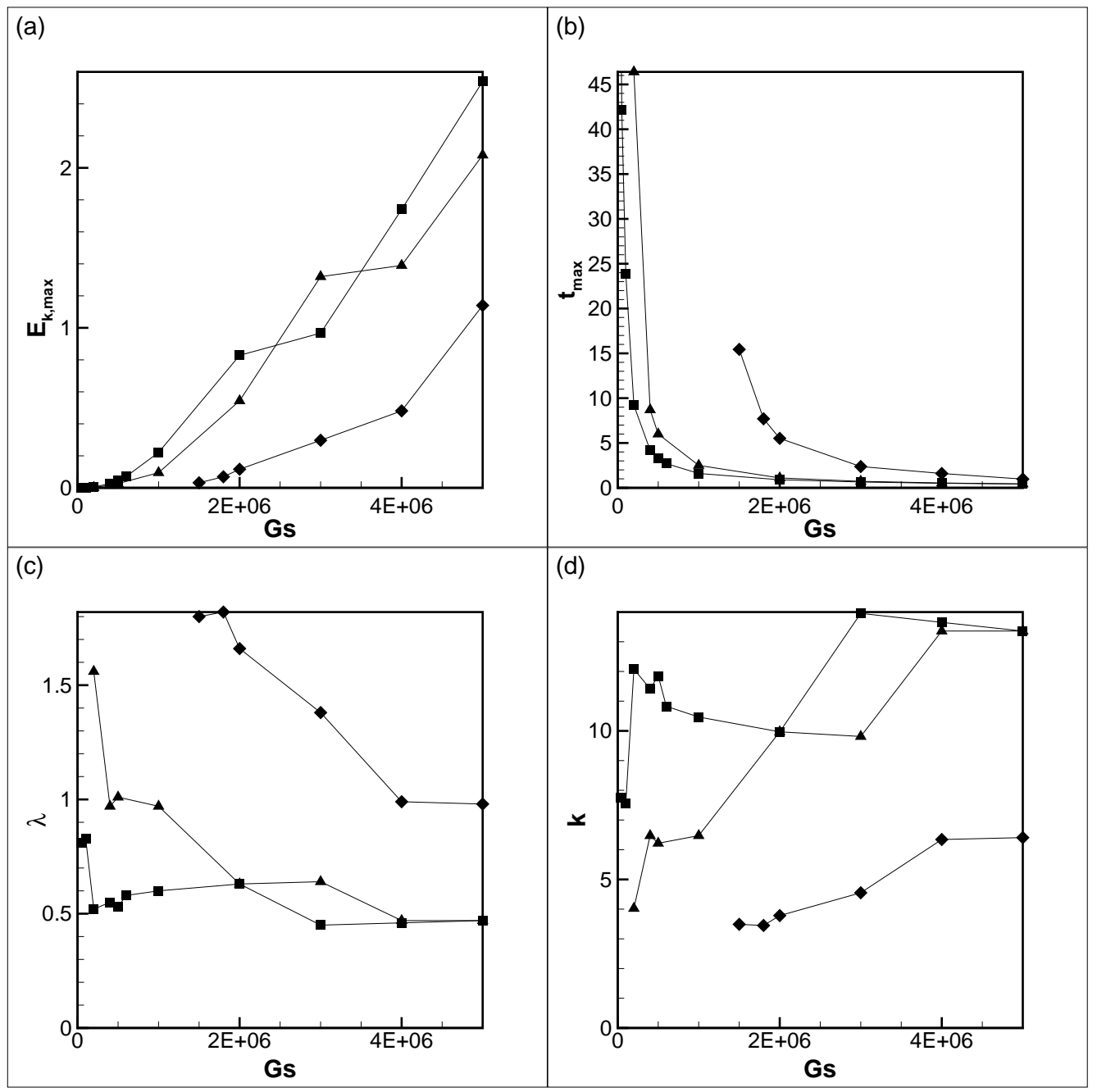

Figure 14. (a,b) The maximum value of the kinetic energy of the average flow and the time moment when the kinetic energy is maximum vs. the Gershuni number. (c,d) The wavelengths and the wavenumbers that characterise the periodicity of the frozen waves vs. the Gershuni number. The data are obtained for the phase-field model, for the parameters $S c=10^{4}$, $C n=2.5 \cdot 10^{-5}, A=-0.5$ and $M=1$ ('squares'), $M=0.001$ ('triangles'), and $M=0.0001$ ('diamonds').

force remains insignificant (and the behaviour of the mixture remains very close to the one predicted by the classical equations) for all $M>0.01$. The clear differences appear when the Mach number is $M=0.001$ and lower. The general dynamics of a mixture always remains similar to the one obtained with the help of the classical approach, however, the threshold Gershuni number required for the ignition of the frozen waves and the levels of vibrations that control the number of the pillars in the container change, which can be seen in Figure 14 and in Table 1.

Figures 15 show the frozen structures that are formed in a container under the critical vibrational forcing for different Mach numbers. All structures look similar, although the lower the value of the Mach number is, the more elastic the interface becomes thus reducing the growth of disturbances with shorter wavelengths. In Figures $15 \mathrm{c}, \mathrm{d}$, there is 


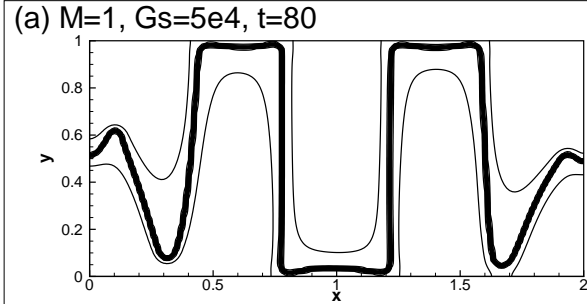

(c) $\mathrm{M}=0.001, \mathrm{Gs}=2 \mathrm{e} 5, \mathrm{t}=55$

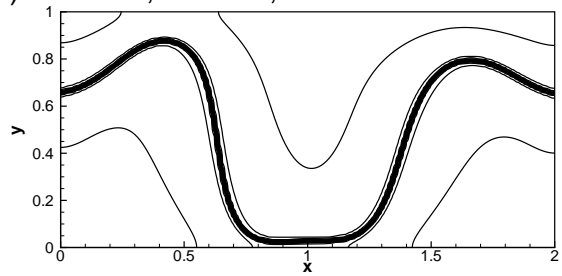

(b) $M=0.01, G s=1 e 5, t=70$

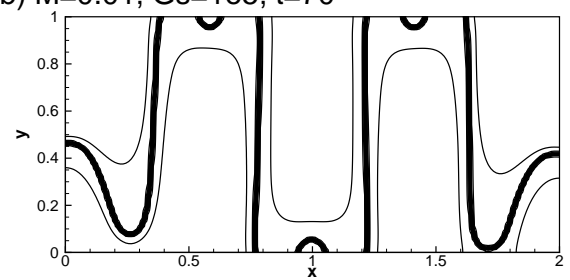

(d) $\mathrm{M}=0.0001, \mathrm{Gs}=2 \mathrm{e} 6, \mathrm{t}=7.2$

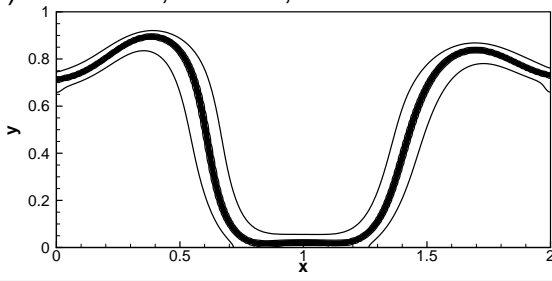

Figure 15. The shape of an interface subjected to the vibrations, for the parameters, (a) $M=1$, $G s=5 \cdot 10^{4}$, (b) $M=0.01, G s=10^{5}$, (c) $M=0.001, G s=2 \cdot 10^{5}$, and $M=0.0001, G s=2 \cdot 10^{6}$ (b). The other parameters are $S c=10^{4}, A=-0.5$, and $C n=2.5 \cdot 10^{-5}$.

$$
\begin{array}{c||c|c|c|c|c}
M & \text { classical } & 1 & 0.01 & 0.001 & 0.0001 \\
G s_{c} & 4 \cdot 10^{4} & 3 \cdot 10^{4} & 10^{5} & 2 \cdot 10^{5} & 1.5 \cdot 10^{6} \\
k_{c} & 7.0 & 7.8 & 7.5 & 4.0 & 3.5 \\
2 \sigma k_{c} S c M^{-1} & 0 & \sim 90 & \sim 9000 & \sim 5 \cdot 10^{4} & \sim 4 \cdot 10^{5}
\end{array}
$$

TABLE 1. The threshold Gershuni numbers, $G s_{c}$, the wavenumbers that characterise the periodicity of the critical frozen waves (that are formed under the critical value of the Gershuni number), $k_{c}$, and the threshold Gershuni numbers predicted by the linear theory vs. the Mach number. The other parameters for the numerical runs are $S c=10^{4}, A=-0.5$, and $C n=2.5 \cdot 10^{-5}$. For the classical approach, $G s_{c}$ determines the level of vibrations when the interface shape becomes primarily determined by diffusion rather than by vibrations.

only one pillar in the container, with the size of nearly 1 , half of the container's length. Such a frozen pattern is the structure with the smallest wavenumber that may be realised in the container. The similar structures were also observed in the experiment (Gaponenko et al. 2015b).

We also want to make some further correlations with the linear theory by Lyubimov \& Cherepanov (1986). The non-dimensional form of equation (1.1) looks as follows

$$
\frac{G s_{c} M}{2 S c}=\sigma k \text {. }
$$

Here, we assumed the weightlessness conditions; $\sigma$ is the non-dimensional coefficient of surface tension that is introduced as $\sigma_{*} /\left(\rho_{*} \mu_{*} L_{*}\right)$, and that is determined by the integral

$$
\sigma=M \frac{E_{i}}{L}=C n \int_{V}(\nabla C)^{2} d V
$$

where $L$ is the length of the interface (for the $2 D$ problem, $L$ plays the role of the surface area of the interface, and $V$ is the surface area of the computational domain). For calculation of the interface length we may use the volume of the interface and the fact that the interface thickness remains almost constant in the phase-field simulations.

For instance, Figure 16 depicts the development of the instability for the different 


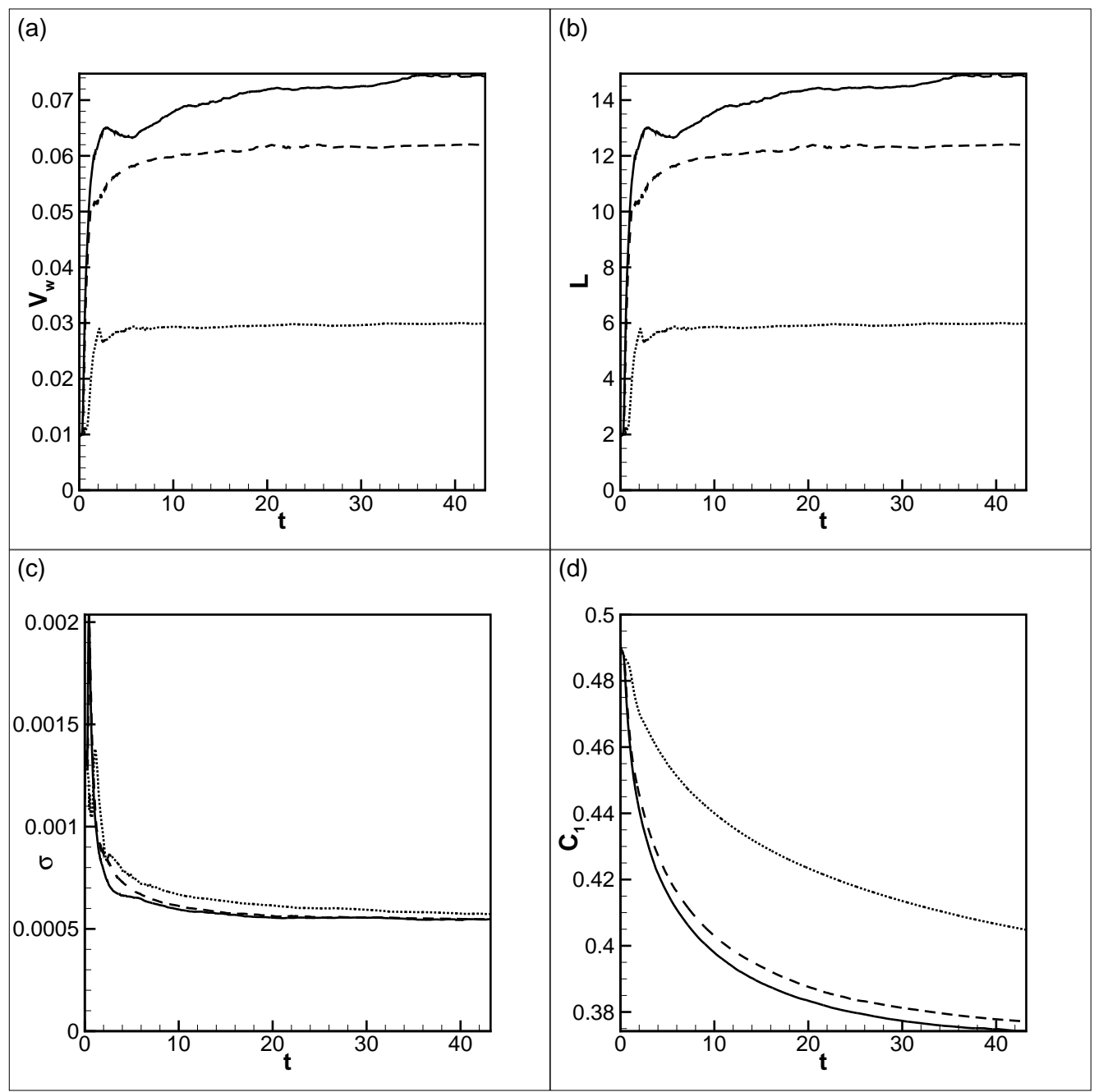

Figure 16. (a) The volume of the interface, (b) the length of the interfacial line, (c) the surface tension coefficient, and (d) the average concentration in liquid 1 vs. time. The data are obtained for the runs fulfilled with the use of the phase-field model for $S c=10^{4}, A=-0.5, C n=2.5 \cdot 10^{-5}$, $G s=5 \cdot 10^{6}$, and $M=1$ (solid line), $M=0.001$ (dashed line), $M=0.0001$ (dotted line).

values of the Mach number. Figures 16a,b show the volume of the interface and the length of the interfacial line, and Figure 16c shows the time changes of the surface tension coefficient, $\sigma$. If we neglect the initial spikes in the values of this coefficient and focus on the moments when the frozen waves are already fully set, we may say that all three curves converge to approximately one value, $\sigma \sim 0.0006$, that is primarily determined by the the Cahn number. The value of the surface tension coefficient allows us to estimate the critical Gershuni numbers as predicted by equation (3.1). These numbers are listed in the last row of Table 1. All these numbers are different from the actual critical Gershuni numbers, which should be explained by the fact that equation (3.1) is obtained for an infinite interface while we examine the dynamics of a mixture enclosed in a container, which restricts the range of the wavenumbers. For the classical theory the instability under lower Gershuni numbers is induced by longwave perturbations, with $\lambda \rightarrow \infty$ and $k \rightarrow 0$ (see e.g. Lyubimova et al. 2017) that cannot be realised within a 

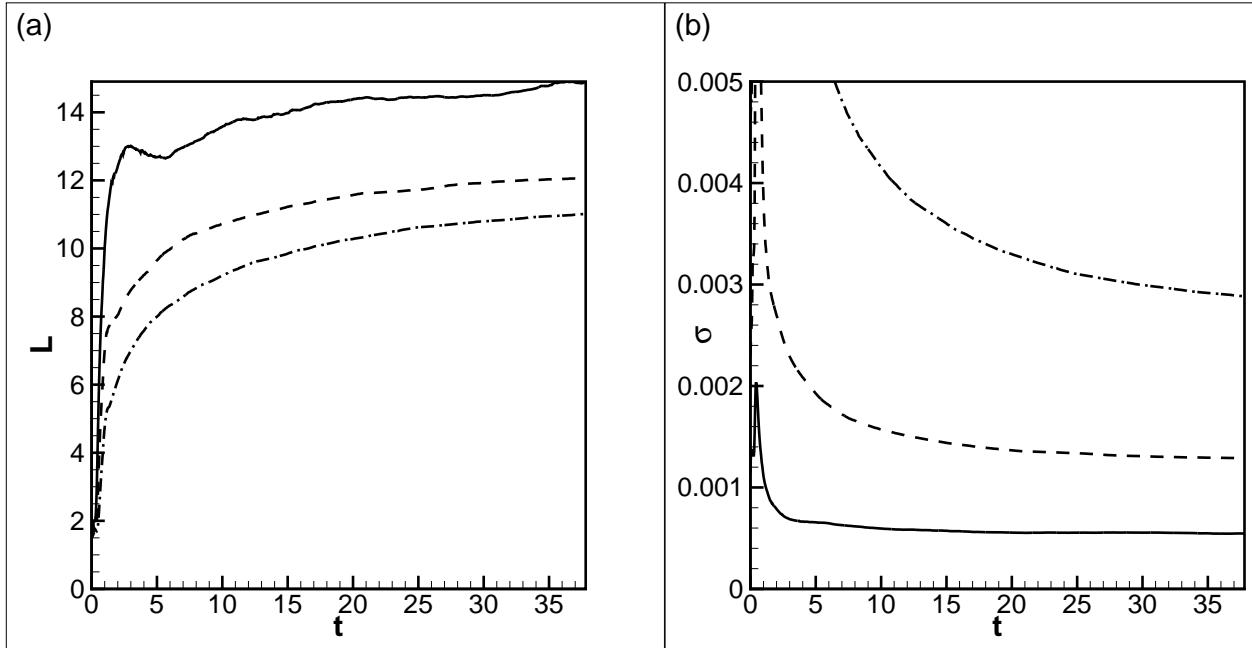

FiguRE 17. (a) The length of the interfacial line, and (d) the surface tension coefficient vs. time. The results are shown for $A=-0.5, S c=10^{4}, G s=5 \cdot 10^{6}, M=1$, and $C n=2.5 \cdot 10^{-5}$ (solid lines), $C n=10^{-4}$ (dashed lines), and $C n=4 \cdot 10^{-4}$ (dash-dotted lines).

confined geometry. Although one sees that when the role of the surface tension forces grows so the wavelength of the critical structure becomes larger, the critical Gershuni numbers predicted by the linear theory (3.1) become closer to the numbers obtained from the numerical simulations.

We also performed the numerical simulations for the different Cahn numbers (the results are depicted in Figure 5, 17 and 18). The change of this parameter does not introduce any qualitative differences to the dynamics of a binary mixture. The change of the Cahn number alters the surface tension coefficient, which is shown in Figure 17, and these changes explain the slight differences in the wave patterns that are formed, as may be observed in Figure 18.

\section{Correlation with the experiment}

We correlate the numerical results with the microgravity experiments that were conducted by Gaponenko et al. $(2015 a, b)$. The experiments were conducted during parabolic flights, so they were characterised by a relatively short durations (the data sets cover only $12.5 \mathrm{~s})$.

The experimental settings set the typical length scale as $L_{*} \approx 8 \cdot 10^{-3} \mathrm{~m}$ (which is the height of the container). The liquid/liquid mixture used for the experiments is characterised by the density contrast, $\phi \approx 0.08$, the density, $\rho_{*} \approx 900 \mathrm{~kg} \mathrm{~m}^{-3}$, the kinematic viscosity, $\left(\eta_{*} / \rho_{*}\right) \approx 2 \cdot 10^{-6} \mathrm{~m}^{2} \mathrm{~s}^{-1}$, and the diffusion coefficient, $D_{*} \approx 4$. $10^{-10} \mathrm{~m}^{2} \mathrm{~s}^{-1}$ (these are the averages of the density, and viscosity and diffusion coefficients for two components of the mixture). These coefficients, in particular, define the following scales of time and velocity, $\tau \sim 30$ s and $u_{*} \sim 3 \cdot 10^{-4} \mathrm{~ms}^{-1}$. Thus, the duration of the experiment is only 0.3 units of the non-dimensional time. The level of vibrations near the reported threshold (see figure 5 in Gaponenko et al. 2015a), but still above the threshold, are set by the amplitude $a \approx 2 \cdot 10^{-3} \mathrm{~m}$ and the angular frequency $\omega \approx 100 \mathrm{~s}^{-1}$ (the vibrational velocity is $a \omega \approx 0.2 \mathrm{~m} \mathrm{~s}^{-1}$ and the vibrational acceleration is $a \omega^{2} \approx 20 \mathrm{~ms}^{-2} \sim 2 g$, where $g$ is the gravity acceleration).

Thus, for this experiment, the non-dimensional parameters of the classical model can 


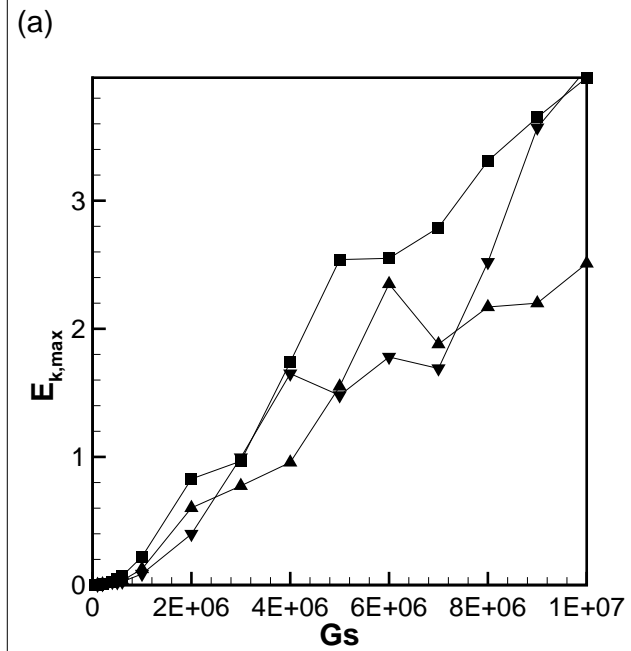

(c)

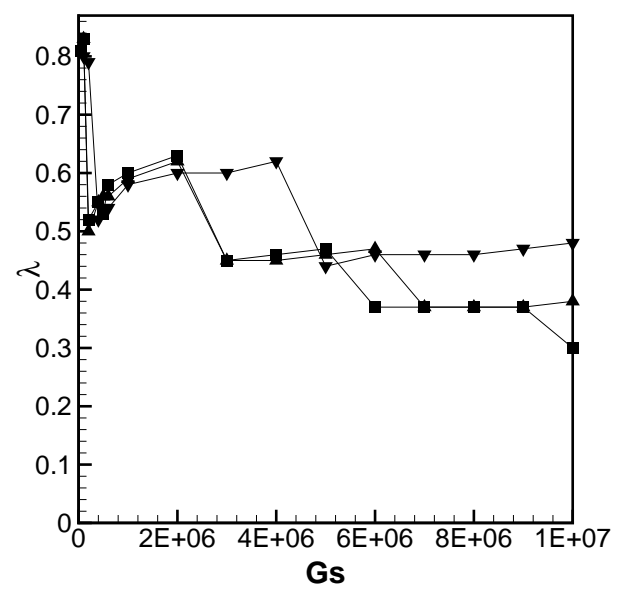

(b)

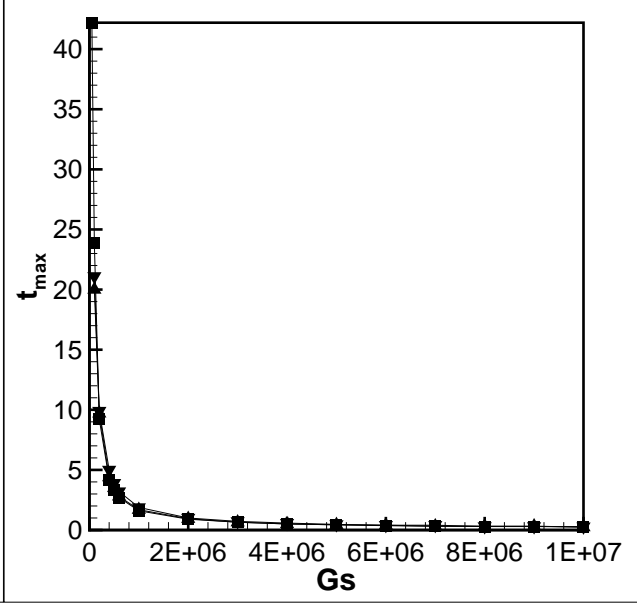

(d)

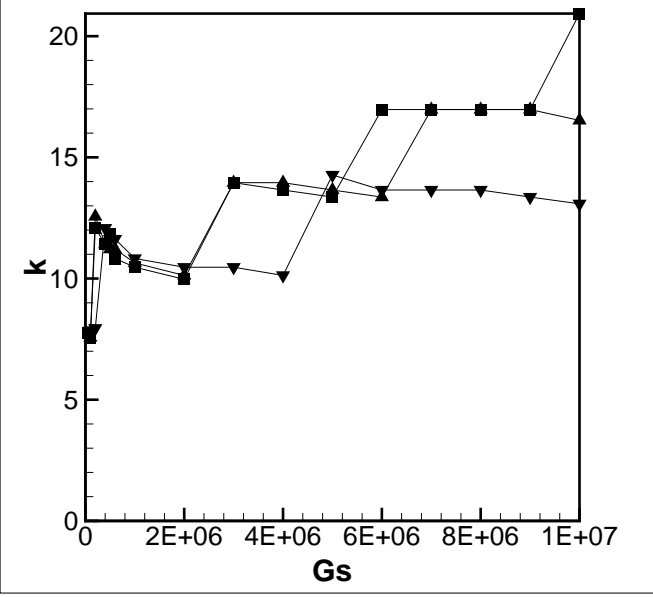

Figure 18. $(\mathrm{a}, \mathrm{b})$ The maximum value of the kinetic energy of the average flow and the time moment when the kinetic energy is maximum vs. the Gershuni number. (c,d) The wavelengths and the wavenumbers that characterise the periodicity of the frozen waves vs. the Gershuni number. The data are obtained for the phase-field model, for the parameters $S c=10^{4}, M=1$, $A=-0.5$ and $C n=2.5 \cdot 10^{-5}$ ('squares'), $C n=10^{-4}$ ('triangles'), and $C n=4 \cdot 10^{-4}$ ('nablas').

be roughly estimated as $S c \sim 5 \cdot 10^{3}$ and $G s_{c} \sim 10^{7}$ (or, $\left.\left(G s_{x} / S c\right) \sim 2000\right)$, which is well above $\left(G s_{c} / S c\right) \sim 1$ that determines the level of vibrations when diffusion starts to dominate over hydrodynamics (below this level the formation of the frozen waves does not occur). One may argue that the non-ignition of the frozen waves under lower levels of vibrations may be explained by a relatively short duration of the microgravity experiments, although, the reported wavelengths that characterise the experimentallyobserved frozen waves are of the order of the cavity length, $\lambda \sim 1$, which indicates the near-the-threshold behaviour. Thus, we conclude that the classical approach cannot describe the threshold generation of the frozen waves.

Correlations of the phase-field results with the experiment is complicated by the use of the non-standard phenomenological parameters. From the definitions of the nondimensional parameters (2.21)-(2.22) we understand that for practical calculations one 
needs to know the mobility coefficient $\alpha$, the capillary constant $\epsilon$, and a typical value of the chemical potential $\mu_{*}$. These parameters are not directly measurable, nevertheless their values may be determined from the following procedure.

First, the mobility coefficient is obviously proportional to the diffusion coefficient, and one may write that $\alpha=\rho_{*} D_{*} / \mu_{*}$.

The capillary constant may be obtained from the measurements of the interface thickness $\epsilon \sim\left(\mu_{*} \delta_{*}\right)^{2}$, (here $\delta_{*}$ is a typical interface thickness). The measurements of the interfacial thickness are quite rare. Molecular dynamics simulations indicate that the thickness of the interface is just several molecular layers, $\sim 1 \mathrm{~nm}$ (see e.g. Jacqmin 2000). Nevertheless, some direct measurements of this parameter can be also found in e.g. the work by Zoltowski et al. (2007), where the thickness of the interface in a monomer/polymer binary mixture is measured as approximately $0.01 \mathrm{~cm}$ (which seems to be the case of a very diffusive interface in a mixture of two fully miscible liquids after their prolonged contact).

Finally, the typical value of the chemical potential can be obtained from the measurements of the surface tension coefficient, since $\mu_{*}=\sigma_{*} /\left(\rho_{*} L_{*} \sigma\right)$, and such measurements for several mixtures are available e.g. in papers by Pojman et al. (2006); Zoltowski et al. (2007). Gaponenko et al. (2015b) also provided the estimation of the surface tension coefficient for the mixture used in their experiments. By correlating the experimental data with the linear theory by Lyubimov \& Cherepanov (1986), they suggested that the miscible interface is to be endowed with the effective surface tension coefficient of $\sigma_{*} \approx 6 \cdot 10^{-6} \mathrm{Nm}^{-1}$. In our calculations the non-dimensional coefficient of the surface tension $\sigma$ depends on the Cahn number, and, as can be seen in Figure 17, this coefficient is roughly equal to $\sigma \sim 6 \cdot 10^{-4}$ for most of our calculations. Combining all these data, we can show that the typical value of the chemical potential is $\mu_{*} \sim 10^{-3} \mathrm{~J} \mathrm{~kg}^{-1}$.

The numerical results obtained on the basis of the phase-field model are sensitive to the value of the Mach number, which can be estimated as $M \sim 6 \cdot 10^{-3}$ for the experiment by Gaponenko et al. $(2015 a, b)$. This is a very rough estimate, but it improves the interpretation of the experimental results that are reported by Gaponenko et al. $(2015 a, b)$. Indeed, Figure 14 and table 1 indicate that the threshold Gershuni number for the estimated level of the surface tension forces (that is set by the Mach number) is roughly to be $G s_{c} \sim 10^{5}$, which is in a more favorable agreement with the experimental parameters, as compared with the predictions of the classical approach. Nevertheless, this threshold level is still substantially below the value reported in the experiment. This can be explained by a substantial slowing down of the development of the frozen wave instability at the near-the-threshold levels of vibrations. By extending the time of the experiment, observations of the frozen waves at lower Gershuni numbers should become possible, making the measured threshold level of vibrations closer to the theoretical prediction.

Another measure to solve the discrepancy of the theory and experiment is to reconsider/improve the estimate of the surface tension coefficient reported by Gaponenko et al. $(2015 b)$. The original estimate is obtained by the comparison of the experimental results with the predictions of the linear theory that deals with inviscid liquids, unbounded interfaces, and the most dangerous but not the fastest growing normal perturbations (the fastest growing modes define the shape of the frozen pattern).

\section{Conclusions}

We study the evolution of a heterogeneous binary mixture that is subjected to highfrequency vibrations. We assume that the initial state of a mixture is different from 
the state of thermodynamic equilibrium, so the hydrodynamic evolution of a mixture is accompanied by the diffusive mass transport. Weightlessness conditions are also assumed. We found that if the level of vibrations exceeds a certain threshold value, then the frozen waves in the form of pillars (or vertical stripes) that stretch from top to bottom of the container (with the walls perpendicular to the direction of vibrations) are formed. The threshold level of vibrations is determined by interfacial stresses that need to be associated even with miscible liquid/liquid boundaries.

The phenomenon of the frozen waves in a mixture of two miscible liquids was earlier studied experimentally and numerically. The numerical modelling was based on the classical approach, that represented a heterogeneous binary mixture as a single-phase fluid with an impurity and that disregarded the surface tension effects for miscible interfaces. This simplified approach produced the results that were in a generally good agreement with the experiments, in terms that the experimentally observed frozen patterns could be well reproduced. However, in accordance with the linear stability theory of an interface subjected to high-frequency vibrations (1.1), the instability should occur unconditionally. This conclusion could be derived from a simple analysis of the governing equations for the classical model, where one could easily see that the vibrational force is the only term capable of setting the concentration field (or the shape of the miscible interface). In the experiment however the start of the instability was characterised by a threshold level of vibrations. To fit the experimental data with formula (1.1) it was earlier proposed to introduce the effective surface tension to be associated with a miscible interface. This effect was missing in the classical model and thus the classical model could not reproduce all phenomena observed in the experiment.

In our work, in addition to the classical approach, we undertake the modelling of the experiment on the basis of the phase-field approach. The particular features of the phase-field approach are the account of the surface tension effects and the more general description of the diffusion process, permitting consideration of mixtures of fully miscible and partially miscible liquids. The governing equations that describe the influence of the high-frequency vibrational forcing on the dynamics of a heterogeneous binary mixture are obtained in the first part of the work.

First, we re-confirm that many experimental features of the frozen wave instability can in fact be reproduced on the basis of the simplified traditional classical approach. The success of the classical model can be explained by the relatively low surface tension forces associated with miscible interfaces, as well as by a sufficiently fast rate of development of the frozen waves which makes diffusion effect insignificant. However, a clear failure of the classical model is an inability to predict the threshold level of vibrations. A particular focus of our work was also to investigate the behavior of miscible liquids under very low level of vibrations, to understand if the classical model can explain the threshold ignition of the frozen waves. We found that under very weak levels of vibrations the classical approach predicts the disappearance of the frozen waves (if the liquids are miscible in all proportions), which is explained by the increase of the diffusion effect (and by associated further weakening of the vibrational forcing due to interface smearing), so the frozen wave instability has no time to develop. Such a behaviour however should be characterised by very diffusive interfacial boundaries that are not observed in the relatively short microgravity experiments.

On the basis of the phase-field theory we reproduce all experimental observations, including the threshold ignition of the instability. We show that the threshold Gershuni number primarily depends on the value of the effective surface tension coefficient that is associated with a miscible boundary. The correlation of the numerical simulations with the microgravity experiment gives a good agreement between the experimentally- 
observed and theoretical-predicted threshold levels of vibrations. In addition, the simulations show that the time needed for setting up the frozen waves increases when the Gershuni number approaches the threshold value. This imposes a substantial restriction on the minimum duration of the experiment. We also observe that upon further increase of the vibrational forcing (which intensity is solely determined by the Gershuni number, i.e. by a square of the vibrational velocity $a \omega$ ) the frozen pattern may change (additional frozen waves appear in the container); the changes of the pattern occur discretely, over certain ranges of the Gershuni number.

In our work we fulfill quite long numerical calculations that describe the evolution of a binary mixture for the period of about 1 hour. The frozen wave patten remain stable during the whole run, no additional instabilities within a transitional layer, that were expected by Wolf (2018), were noticed. Depending on whether the liquids are fully or partially miscible, the pillar's walls either smear until they become completely indistinguishable, or remain sharp indefinitely long. In the latter case the diffusion process ends when the concentrations in the neighbouring components reach the equilibrium values, so the binary mixture attains the state of mechanical and thermodynamic equilibrium.

In conclusion, we would like to state that the time-average equations, that are derived in the first part of our work, present a powerful computational tool that allows to trace the dynamics of miscible (completely or partially miscible) and immiscible liquids with the correct account of the surface tension effects that needs to be associated even with miscible interfaces, and which is not possible to do on the basis of the classical approach traditionally used for the description of miscible interfaces.

\section{REFERENCES}

Ahmadlouydarab, M. \& Feng, J. J. 2014 Motion and coalescence of sessile drops driven by substrate wetting gradient and external flow. J. Fluid Mech. 746, 214-235.

Bezdenezhnyh, N. A., Briskman, V. A., Lapin, A. Y., Lyubimov, D. V., Lyubimova, T. P., Tcherepanov, A. A. \& Zakharov, I. A. 1991 The influence of high frequency tangential vibrations on the stability of the fluid interface in microgravity. Int. J. for Microgravity Research and Applications. 4, 96-97.

Ding, H., Spelt, P.D.M. \& Shu, C. 2007 Diffuse interface model for incompressible two-phase flows with large density ratios. Journal of Computational Physics. 226, 2078-2095.

Gandikota, G., Chatain, D., Amiroudine, S., Lyubimova, T. \& Beysens, D. $2014 a$ Frozenwave instability in near-critical hydrogen subjected to horizontal vibration under various gravity fields. Phys. Rev E. 89, 012309.

Gandikota, G., Chatain, D., Lyubimova, T. \& Beysens, D. $2014 b$ Dynamic equilibrium under vibrations of $h_{2}$ liquid-vapor interface at various gravity levels. Phys. Rev E. 89, 063003.

Gaponenko, Y., Torregrosa, M. M., Yasnou, V., Mialdun, A. \& Shevtsova, V. $2015 a$ Interfacial pattern selection in miscible liquids under vibration. Soft Matter. 11, 82218224 .

Gaponenko, Y. A. \& Shevtsova, V. 2010 Effects of vibrations on dynamics of miscible liquids. Acta Astronautica. 66, 174-182.

Gaponenko, Y. A., Torregrosa, M., Yasnou, V., Mialdun, A. \& Shevtsova, V. $2015 b$ Dynamics of the interface between miscible liquids subjected to horizontal vibration. J. Fluid Mech. 784, 342-372.

Gaponenko, Y. A., Volpert, V. A., Zen'kovskaya, S. M. \& Pojman, D. A. 2006 Effect of high-frequency vibration on convection in miscible fluids. J. Fluid Mech. 47, 190-198.

JACQMin, D. 1999 Calculation of two-phase navierstokes flows using phase-field modeling. J. Comput. Phys. 155.

JACQMin, D. 2000 Contact-line dynamics of a diffuse fluid interface. Journal of Fluid Mechanics 402, $57-88$. 
Joseph, D. D. \& Renardy, Y. Y. 1993 Fundamentals of two-fluid dynamics. Part II: lubricated transport, drops and miscible liquids. Springer-Verlag.

Landau, L. D. \& Lifshitz, E.M. 2010 Statistical Physics. Volume 5 of Course of Theoretical Physics.. Elsevier, Butterworth Heinemann.

Lowengrub, J. \& Truskinovsky, L. 1998 Quasi-incompressible cahn-hilliard fluids and topological transitions. Proc. R. Soc. London, Ser. A 454 (2), 2617-2654.

Lyubimov, D. V. \& Cherepanov, A. A. 1986 Development of a steady relief at the interface of fluids in a vibrational field. Fluid Dynamics. 6, 8-13.

Lyubimov, D. V., Ivantsov, A. O., Lyubimova, T. P. \& Khilko, G. L. 2016 Numerical modeling of frozen wave instability in fluids with high viscosity contrast. Fluid Dyn. Res. 48, 61415 .

Lyubimov, D. V., Khilko, G. L., Ivantsov, A. O. \& Lyubimova, T. P. 2017 Viscosity effect on the longwave instability of a fluid interface subjected to horizontal vibrations. J. Fluid Mech. 814, 24-41.

Lyubimov, D. V., Lyubimova, T. P. \& Cherepanov, A. A. 2003 Dynamics of interfaces subject to vibrations. FizMatLit.

Lyubimova, T., Ivantsov, A., Garrabos, Y., Lecoutre, C., Gandikota, G. \& Beysens, D. 2017 Band instability in near-critical fluids subjected to vibration under weightlessness. Phys. Rev. E. 95, 013105.

Lyubimova, T., Vorobev, A. \& Prokopev, S. 2019 Rayleigh-taylor instability of a miscible interface in a confined domain. Physics of Fluids 31, 014104.

Pojman, J. A., Whitmore, C., Liveri, M. L. T., Lombardo, R., Marszalek, J., Parker, R. \& Zoltowski, B. 2006 Evidence for the existence of an effective interfacial tension between miscible fluids: isobutyric acidwater and 1-butanolwater in a spinning-drop tensiometer. Langmuir. 22, 2569-2577.

Prokopev, S, Vorobev, A. \& Lyubimova, T. 2019 Phase-field modeling of an immiscible liquid-liquid displacement in a capillary. Phys. Rev. E 99, 033113.

Shevtsova, V., Gaponenko, Y., Yasnou, V., Mialdun, A. \& Nepomnyashchy, A. 2015 Wall-generated pattern on a periodically excited miscible liquid/liquid interface. Langmuir. 31, 5550-5553.

Shevtsova, V., Gaponenko, Y. A., Yasnou, V., Mialdun, A. \& Nepomnyashchy, A. 2016 Two-scale wave patterns on a periodically excited miscible liquid-liquid interface. J. Fluid Mech. 795, 409-422.

Stevar, M. S. P. \& Vorobev, A. 2012 Shapes and dynamics of miscible liquid/liquid interfaces in horizontal capillary tubes. J. Colloid Interface Sci. 383, 184-197.

Talib, E., Jalikop, S. V. \& Juel, A. 2007 The influence of viscosity on the frozen wave instability: theory and experiment. J. Fluid Mech. 584, 45-68.

Vorobev, A. 2014 Dissolution dynamics of miscible liquid/liquid interfaces. Curr. Opinion Colloid Interface Sci. 19, 300-308.

Vorobev, A. \& Boghi, A. 2016 Phase-field modelling of a miscible system in spinning droplet tensiometer. J. Colloid Interface Sci. 482, 193-204.

Vorobev, A. \& Khlebnikova, E. 2018 Modelling of the rise and adsorption of a fluid inclusion. Int. J. Heat Mass Trans. 125, 801-814.

Wolf, G. H. 1961 The dynamic stabilization of the rayleigh-taylor instability an the corresponding dynamic equilibrium. Z. Physik. 227, 291-300.

Wolf, G. H. 1970 Dynamic stabilization of the interchange instability of a liquid-gas interface. Phys. Rev. Lett. 24, 444-446.

Wolf, G. H. 2018 Dynamic stabilization of the rayleigh-taylor instability of miscible liquids and the related "frozen waves". Physics of Fluids. 30, 021701.

Xie, R. \& Vorobev, A. 2016 On the phase-field modelling of a miscible liquid/liquid boundary. J. Colloid Interface Sci. 464, 48-58.

Yoshikawa, H. N. \& Wesfreid, J. E. 2011a Oscillatory kelvin-helmholtz instability. part 1. a viscous theory. J. Fluid Mech. 675, 223-248.

Yoshikawa, H. N. \& Wesfreid, J. E. $2011 b$ Oscillatory kelvin-helmholtz instability. part 2. an experiment in fluids with a large viscosity contrast. J. Fluid Mech. 675, 249-267.

Zoltowski, B., Chekanov, Y., Masere, J., Pojman, J. A. \& Volpert, V. 2007 Evidence 
for the existence of an effective interfacial tension between miscible fluids. 2. dodecyl acrylatepoly(dodecyl acrylate) in a spinning drop tensiometer. Langmuir. 23, 5522-5531. 